\title{
Temporal evolution of the Evershed flow in sunspots ${ }^{\star}$
}

\section{Observational characterization of Evershed clouds}

\author{
D. Cabrera Solana ${ }^{1}$, L. R. Bellot Rubio ${ }^{1}$, C. Beck ${ }^{2}$, and J. C. del Toro Iniesta ${ }^{1}$ \\ 1 Instituto de Astrofísica de Andalucía, CSIC, Apdo. 3004, 18080 Granada, Spain \\ e-mail: 1bellot@iaa.es \\ 2 Instituto de Astrofísica de Canarias, C/Via Láctea s/n, 38200 La Laguna, Tenerife, Spain
}

Received 8 May 2007 / Accepted 19 July 2007

\begin{abstract}
Context. The magnetic and kinematic properties of the photospheric Evershed flow are relatively well known, but not completely understood. The evolution of the flow with time, which is mainly due to the appearance of velocity packets called Evershed clouds (ECs), may provide information to further constrain its origin.

Aims. We undertake a detailed analysis of the evolution of the Evershed flow by studying the properties of ECs. In this first paper we determine the sizes, proper motions, location in the penumbra, and frequency of appearance of ECs, as well as their typical Doppler velocities, linear and circular polarization signals, Stokes $V$ area asymmetries, and continuum intensities.

Methods. High-cadence, high-resolution, full vector spectropolarimetric measurements in visible and infrared lines are used to characterize the EC phenomenon through a simple line-parameter analysis.

Results. ECs appear in the mid penumbra and propagate outward along filaments having large linear polarization signals and enhanced Evershed flows. The frequency of appearance of ECs varies between 15 and $40 \mathrm{~min}$ in different filaments. ECs exhibit the largest Doppler velocities and linear-to-circular polarization ratios of the whole penumbra. In addition, lines formed deeper in the atmosphere show larger Doppler velocities, much in the same way as the "quiescent" Evershed flow. According to our observations, ECs can be classified in two groups: type I ECs, which vanish in the outer penumbra, and type II ECs, which cross the outer penumbral boundary and enter the sunspot moat. Most of the observed ECs belong to type I. On average, type II ECs can be detected as velocity structures outside of the spot for only about $14 \mathrm{~min}$. Their proper motions in the moat are significantly reduced with respect to the ones they had in the penumbra.
\end{abstract}

Key words. sunspots - magnetic fields - Sun: photosphere

\section{Introduction}

The Evershed flow has been studied intensively over the last decades (for a review see, e.g., Solanki 2003). Our understanding of this dynamical phenomenon is based on observations that do not reflect its temporal variability. The study of the evolution of the flow requires stable observing conditions during long periods of time, which is difficult without adaptive optics systems. It is known, however, that the Evershed flow displays variations on time scales of 8-25 min due to the appearance of packets of enhanced Doppler signals (Shine et al. 1994; Rimmele 1994; Rouppe van der Voort 2003; Georgakilas \& Christopoulou 2003). These packets, called Evershed clouds (ECs), move from the mid penumbra to the outer penumbral boundary where they disappear.

A good knowledge of ECs is crucial for a complete understanding of the nature of the Evershed flow. Previous investigations were based on high resolution filtergrams and Dopplergrams, but the polarization signals of ECs have never been observed. As a result, important details such as the relation between magnetic fields and ECs remain unknown.

In a series of two papers we present the first study on the temporal evolution of the Evershed flow that makes use

^ Appendices A and B are only available in electronic form at http://www . aanda. org of high-cadence, high-precision, full vector spectropolarimetric observations of visible and near-infrared lines. The spatial resolution of these measurements, around $0 .{ }^{\prime} 6$ at $1565 \mathrm{~nm}$ and 0.7 at $630 \mathrm{~nm}$, is one of the highest ever achieved in solar spectropolarimetry.

This first paper concentrates on the observational characterization of ECs. In Sect. 2 we describe the observations and data reduction. We then identify the ECs and determine their lifetimes, proper motions, and sizes (Sect. 3). Sections 4 and 5 investigate the properties of the penumbral filaments along which ECs move, and the periodicity of the EC phenomenon. We find that ECs occur in the center of intra-spines, i.e., elongated structures having the more horizontal fields and stronger Evershed flows of the penumbra (Lites et al. 1993). The spectroscopic and polarimetric properties of the ECs are presented in Sect. 6, and compared with those of the intra-spines in Sect. 7. The dependence of the Doppler velocities on height is examined in Sect. 8. We also study the correlation between proper motions and Doppler velocities (Sect. 9), the evolution of the properties of the ECs as they propagate outward (Sect. 10), and their disappearance inside (Sect. 11) and outside (Sect. 12) the penumbra. Our main findings are summarized in Sect. 13.

Paper II of the series (Cabrera Solana et al. 2007) contains an analysis of the thermal, magnetic, and dynamic properties of the ECs as inferred from inversions of the measured Stokes spectra. Further details can be found in Cabrera Solana (2007). 
Table 1. Visible and infrared spectral lines observed with POLIS and TIP. $\lambda_{0}$ represents the laboratory central wavelength, $\chi$ the excitation potential, and $\log g f$ the logarithm of the oscillator strength times the multiplicity of the lower level. $a, b$, and $c$ refer to Nave et al. (1994), Borrero et al. (2003), and Pierce \& Breckenridge (1974), respectively. Note that $\mathrm{O}_{2}$ are telluric absorption lines.

\begin{tabular}{lccccc}
\hline \hline Instrument & Species & $\begin{array}{c}\lambda_{0} \\
{[\mathrm{~nm}]}\end{array}$ & $\begin{array}{c}\chi \\
{[\mathrm{eV}]}\end{array}$ & $\log g f$ & transition \\
\hline POLIS & $\mathrm{Fe} \mathrm{I}$ & $630.1501^{a}$ & $3.65^{a}$ & -0.72 & ${ }^{5} P_{2}-{ }^{5} D_{2}$ \\
POLIS & $\mathrm{Fe} \mathrm{I}$ & $630.2494^{a}$ & $3.69^{a}$ & -1.24 & ${ }^{5} P_{1}-{ }^{5} D_{0}$ \\
POLIS & $\mathrm{Fe} \mathrm{I}$ & 630.3460 & 4.32 & -2.55 & ${ }^{5} G_{6}-{ }^{5} G_{5}$ \\
POLIS & $\mathrm{Ti} \mathrm{I}$ & 630.3753 & 1.45 & -1.44 & ${ }^{3} F_{3}-{ }^{3} G_{3}$ \\
POLIS & $\mathrm{O}_{2}$ & $630.2001^{c}$ & & & \\
POLIS & $\mathrm{O}_{2}$ & $630.2761^{c}$ & & & \\
TIP & $\mathrm{Fe} \mathrm{I}$ & $1564.7410^{a}$ & $6.33^{a}$ & -0.95 & ${ }^{7} D_{1}-{ }^{5} P_{2}$ \\
TIP & $\mathrm{Fe} \mathrm{I}$ & $1564.8515^{a}$ & $5.43^{a}$ & $-0.68^{b}$ & ${ }^{7} D_{1}-{ }^{7} D_{1}$ \\
TIP & $\mathrm{Fe} \mathrm{I}$ & $1565.2874^{a}$ & $6.25^{a}$ & $-0.05^{b}$ & ${ }^{7} D_{5}-{ }^{6} D_{4.5} 4 f[3.5]_{4}^{0}$ \\
\hline
\end{tabular}

Table 2. Log of the VTT observations. The second column gives the heliocentric angle of the spot. The third and fourth columns show the scanning step and the integration time per slit position. The duration of the sequences and the seeing conditions are indicated in the last two columns.

\begin{tabular}{cccccc}
\hline \hline Date & $\begin{array}{c}\theta \\
{[\mathrm{deg}]}\end{array}$ & $\begin{array}{c}\text { step size } \\
{[\operatorname{arcsec}]}\end{array}$ & $\begin{array}{c}t_{\mathrm{i}} \\
{[\mathrm{s}]}\end{array}$ & $\begin{array}{c}\text { duration } \\
{[\mathrm{min}]}\end{array}$ & $\begin{array}{c}\text { seeing } \\
{[\operatorname{arcsec}]}\end{array}$ \\
\hline $06 / 30 / 2005$ & 43 & 0.2 & 10.5 & 156 & $\sim 0.6-0.7$ \\
$07 / 01 / 2005$ & 35 & 0.2 & 5.5 & 80 & $\sim 0.8-0.9$ \\
\hline
\end{tabular}

\section{Observations and data analysis}

\subsection{Observations}

NOAA Active Region 10781 was observed on 2005 June 30 from 8:47 to 11:23 UT and on 2005 July 1 from 9:31 to 10:51 UT at the German Vacuum Tower Telescope (VTT) on Tenerife. The Tenerife Infrared Polarimeter (TIP; Martínez Pillet et al. 1999; Collados et al. 1999) and the Polarimetric Littrow Spectrograph (POLIS; Schmidt et al. 2003; Beck et al. 2005b) were operated simultaneously to record the full Stokes profiles of the spectral lines around $1565 \mathrm{~nm}$ and $630 \mathrm{~nm}$ (Table 1). The same spot was observed on 2005 June 30 with the Dutch Open Telescope (DOT) from 8:45 to 9:38 UT. Table 2 gives details of the observations at the VTT. The active region was located at heliocentric angles of $43^{\circ}$ and $35^{\circ}$ on June 30 and July 1 , respectively. The slit width/pixel size were $0.36 / 0 . ' 175$ for TIP and $0 . ' 18 / 0$.' $^{\prime} 145$ for POLIS. During the observations, the Kiepenheuer Adaptive Optics System (KAOS; Soltau et al. 2002) was used to reduce image motion and blurring. In order to observe the same field of view (FOV) the TIP and POLIS slits were aligned by rotating the main spectrograph of the VTT and moving the TIP camera.

On June 30, we performed rapid scans of a small portion of the center-side penumbra of the spot including the adjacent moat region (see Fig. 1). The scan step was 0.'2 for a total of 20 steps $\left(4^{\prime \prime}\right)$. The integration time was $10.5 \mathrm{~s}$ per slit position, resulting in a cadence of $3.9 \mathrm{~min}$. The scan was repeated 40 times. The excellent seeing conditions, together with KAOS, allowed us to reach a spatial resolution of about $0 .{ }^{\prime} 6$ at $1565 \mathrm{~nm}$ and 0.7 at $630 \mathrm{~nm}$. These values have been derived from the power spectra of the quiet sun granulation in the vicinity of the spot.

On July 1 we observed a smaller portion of the center-side penumbra of AR 10781: maps 2" -wide were produced with 10 steps of 0.2 . The integration time was $5.5 \mathrm{~s}$ per slit position

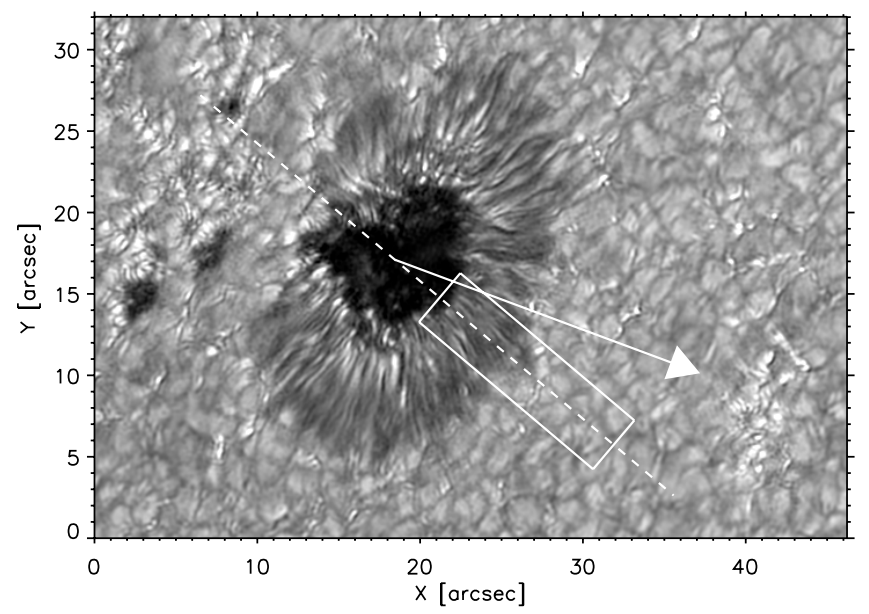

Fig. 1. Speckle-reconstructed G-band filtergram of AR 10781 taken at the DOT on 2005 June 30, 9:34 UT. The box shows the fraction of the center-side penumbra and adjacent moat scanned by TIP and POLIS. The arrow marks the direction to disk center.

( 2 min cadence). The spatial resolution of the July 1 observations is around 0 . $^{\prime} 8$ in the infrared and 0.' 9 in the visible.

The use of infrared (IR) and visible (VIS) spectral lines, with different heights of formation, allows us to obtain physical information from a wide range of layers in the atmosphere. Moreover, the different sensitivities of these lines to the atmospheric parameters improve the diagnostic capabilities of a single spectral range alone (Cabrera Solana et al. 2005).

\subsection{Data reduction}

\subsubsection{Calibration of the polarimetric data}

To remove the different responses of the CCD pixels to homogeneous illumination we applied gain tables calculated from flat field and dark current images. Then, the intensity signals measured by the polarimeters were converted to Stokes profiles using demodulation matrices derived with the help of the calibration optics of the VTT. Instrumental crosstalk was removed applying the telescope model of Beck et al. (2005a), while residual crosstalk due to the coelostat mirrors was corrected for using the statistical method of Collados (2003) and Schlichenmaier $\&$ Collados (2002). The accuracy of the polarization profiles is estimated to be of the order of $10^{-3}$ in units of the continuum intensity (Beck et al. 2005a).

\subsubsection{Scattered light correction}

The presence of residual $\mathrm{O}_{2}$ telluric lines in the polarization profiles recorded by POLIS (Fig. A.2) reveals the existence of spectrally undispersed scattered light. This contamination might be due to incomplete shielding of the light path inside the compact POLIS shelter.

We correct the spectra for scattered light in the same way as Allende Prieto et al. (2004). The amount of scattered light and the resolving power of the polarimeter are calculated by comparing the average quiet Sun intensity profiles measured with POLIS and the Fourier Transform Spectrometer (FTS). The FTS is not affected by scattered light and its point spread function (PSF) can be considered as a Dirac delta function for our purposes. Hence, we should be able to reproduce the observed line shapes by adding a certain fraction of scattered light to the FTS 
Table 3. Mean rms fluctuations of the Doppler shifts in the umbral pixels used to define the zero point of the velocity scale. The errors represent the standard deviations of the averages.

\begin{tabular}{cccccc}
\hline \hline Date & $\begin{array}{c}\sigma_{630.15} \\
{\left[\mathrm{~m} \mathrm{~s}^{-1}\right]}\end{array}$ & $\begin{array}{c}\sigma_{630.25} \\
{\left[\mathrm{~m} \mathrm{~s}^{-1}\right]}\end{array}$ & $\begin{array}{c}\sigma_{630.35} \\
{\left[\mathrm{~m} \mathrm{~s}^{-1}\right]}\end{array}$ & $\begin{array}{c}\sigma_{1564.85} \\
{\left[\mathrm{~m} \mathrm{~s}^{-1}\right]}\end{array}$ & $\begin{array}{c}\sigma_{1565.29} \\
{\left[\mathrm{~m} \mathrm{~s}^{-1}\right]}\end{array}$ \\
\hline $06 / 30$ & $83 \pm 16$ & $80 \pm 17$ & $100 \pm 20$ & $86 \pm 16$ & $120 \pm 16$ \\
$07 / 01$ & $64 \pm 30$ & $63 \pm 31$ & $74 \pm 34$ & $64 \pm 28$ & $60 \pm 28$ \\
\hline
\end{tabular}

profiles and convolving them with the spectral PSF of the polarimeter, i.e.,

$I=\left(\frac{I_{\mathrm{FTS}}+K}{1+K}\right) * G(\sigma)$,

where $I$ are the POLIS disk-center profiles, $I_{\text {FTS }}$ the FTS profiles, $K$ is the fraction of scattered light, $G$ the polarimeter PSF (assumed to be a Gaussian of width $\sigma$ ), and $*$ represents convolution.

Figure A.1 displays an example of the fits obtained using Eq. (1). The best results are achieved with $K \simeq 15 \%$ and $\sigma \simeq 6 \mathrm{pm}$, both for the July 1 and June 30 data sets. The theoretical resolving power of POLIS ( $2 \mathrm{pm}$, limited by the slit) is always smaller than that inferred from the fits. The somewhat large value of $\sigma$ may be a consequence of imperfect focusing of the instrument.

\subsection{Wavelength scale}

The velocity scales for the IR and VIS lines have been set assuming that the umbra is at rest (?). We use the mean position of the Stokes $V$ zero-crossings in the darkest regions of the umbra to define the zero point of the velocity scale ${ }^{1}$. For the June 30 time sequence, we select pixels with continuum intensities $I / I_{\mathrm{qs}}<0.4$ at $630 \mathrm{~nm}$. The dispersion is calculated from the number of pixels between the line core positions of Fe I $1564.85 \mathrm{~nm}$ and Fe I $1565.52 \mathrm{~nm}$ for TIP and the $\mathrm{O}_{2}$ telluric lines for POLIS.

To provide a lower limit for the calibration uncertainty, we compute the rms fluctuation of the Doppler shifts in the selected umbral pixels. This is done for each map. Table 3 shows the average values and their standard deviations for the different lines. As can be seen, the mean fluctuations are always smaller than $120 \mathrm{~m} \mathrm{~s}^{-1}$, with low scatter between maps $\left(<34 \mathrm{~m} \mathrm{~s}^{-1}\right)$. Similar fluctuations have been reported by Rezaei et al. (2006). Most probably, they simply reflect the non-zero velocities of umbral dots (Socas-Navarro et al. 2004) and/or the presence of oscillations in the selected areas.

\subsection{Alignment of TIP, POLIS, and DOT data}

\subsubsection{Alignment of TIP and POLIS}

Since the refractive index of the air depends on wavelength (Reardon 2006), the images of the Sun taken by TIP and POLIS are shifted with respect to each other. In order to remove these shifts, we follow the method described by Beck et al. (2007). It consists in performing a cross-correlation of the intensity maps using the largest pixel (the TIP pixel) as a reference. To that aim, the POLIS data are rebinned to the TIP pixel size. Figure 2 displays, as an example, continuum intensity maps at $1565 \mathrm{~nm}$ and

1 The small focal length of POLIS produces a curvature of the spectrum along the slit. In order to remove it, we used the $\mathrm{O}_{2}$ telluric line at $630.20 \mathrm{~nm}$ to align all the spectra individually. This correction was applied before the determination of the wavelength scale.

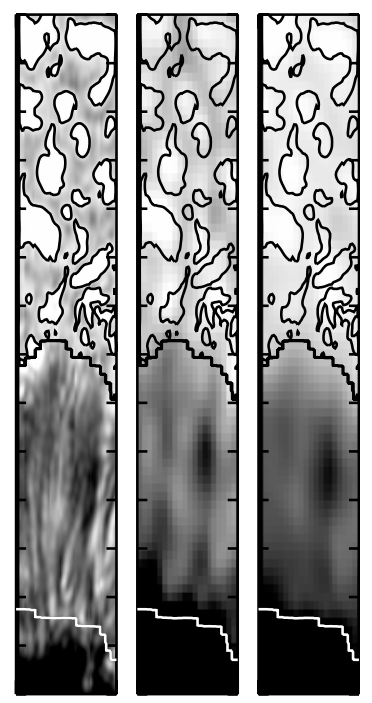

Fig. 2. From left to right: Maps of speckle-reconstructed G-band intensity, continuum intensity at $1565 \mathrm{~nm}$, and continuum intensity at $630 \mathrm{~nm}$ observed on June 30, 9:26 UT. Contours outline pixels with G-band intensities greater than $0.5 I_{\mathrm{qs}}$. Each tickmark on the $y$-axis represents $1^{\prime \prime}$.

$630 \mathrm{~nm}$ after removing the shifts. As can be seen, the correspondence between the intensity structures is quite precise. The accuracy of the alignment between TIP and POLIS is around $0{ }^{\prime} 1$ (Beck et al. 2007).

The position of the Sun on the sky changes during the day. Hence, the differential refraction produces time-dependent shifts between the VIS and IR spectropolarimetric data. This is why we calculate them for each repetition of the scan. The total shifts accumulated during the 156 min covered by the June 30 observations amount to $1^{\prime \prime}$ and $0{ }^{\prime} 6$ in the directions perpendicular and parallel to the slit, respectively. This means that co-spatial Stokes profiles in the visible and the near-infrared are not strictly simultaneous, but the time difference is always smaller than $\sim 55 \mathrm{~s}$.

\subsubsection{Alignment of VTT and DOT data}

Filtergrams and spectropolarimetric maps differ significantly in their nature. The DOT filtergrams represent 2D snapshots, while the VTT maps were constructed by scanning the spectrograph slit accross the solar surface. If one wants to compare both kinds of observations, the positions observed by the polarimeters have to be located on the DOT filtergrams taken at the same time. Following Beck et al. (2007), we align the POLIS slit-jaw images with the DOT G-band filtergrams. This is done by degrading the G-band data to the pixel size of the slit-jaw images. After the rotation between the images of the two telescopes is removed, the shifts between the slit-jaw and the G-band images can be determined by cross-correlation. At this point, one knows the positions of the POLIS/TIP slits on the G-band images. Finally, we construct artificial G-band maps by selecting the co-spatial G-band strip closer in time to the polarimetric data. The DOT and VTT observations are not strictly co-temporal, but the differences are small enough $(\leq 30 \mathrm{~s})$ to work with both data sets as if they had been taken simultaneously.

\subsection{Line parameters}

The simplest way to analyze the observations is by computing line parameters from the polarization profiles. Those parameters reflect the atmospheric conditions of the solar photosphere, 
providing a first estimate of their values. We have derived the following observables from the Stokes profiles of the IR and VIS lines:

1. Continuum intensities, I. According to the EddingtonBarbier approximation, $I$ depends on the temperature of the layers where the continuum is formed $(\tau \sim 1)$. This approximation works well in sunspot penumbrae (del Toro Iniesta et al. 1994; Bellot Rubio et al. 2006).

2. Stokes $V$ zero-crossing velocity. The velocity is estimated from the Doppler shift of the Stokes $V$ zero-crossing wavelength. We use the mid-point between the maxima of the Stokes $V$ lobes to locate the zero crossing. This velocity represents the motion of magnetized plasma in the resolution element and can only be computed for pixels with normal two-lobed Stokes $V$ profiles.

3. Total polarization signals. For fully split lines, if the magnetic field orientation remains constant along the LOS (Solanki 1993):

- The total linear polarization, $L=\sum_{i} \sqrt{Q\left(\lambda_{i}\right)^{2}+U\left(\lambda_{i}\right)^{2}}$, is proportional to $\sin ^{2} \gamma_{\text {LOS }}$. Hence, the larger the value of $L$, the greater the inclination of the magnetic field to the LOS $\left(\gamma_{\mathrm{LOS}}\right)$.

- The total circular polarization, $V=\sum_{i}\left|V\left(\lambda_{i}\right)\right|$, varies as $\cos \gamma_{\text {LOS }}$. Therefore $V$ is smaller when the field inclination is larger.

- The linear-to-circular polarization ratio increases with the field inclination as $L / V \sim \sin ^{2} \gamma_{\mathrm{LOS}} / \cos \gamma_{\mathrm{LOS}}$.

The polarization signals provide only a rough estimate of $\gamma_{\mathrm{LOS}}$, because the magnetic field is known to vary with height in the solar photosphere and the spectral lines are not always in the strong field regime.

4. The Stokes $V$ area asymmetry,

$\delta A=\frac{\left|A_{r}\right|-\left|A_{b}\right|}{\left|A_{r}\right|+\left|A_{b}\right|}$

where $\left|A_{r}\right|$ and $\left|A_{b}\right|$ stand for the areas of the red and blue lobes of Stokes $V$, respectively. $\delta A$ arises as a consequence of velocity gradients along the LOS (Auer \& Heasley 1978; Sánchez Almeida \& Lites 1992). The combination of such gradients with magnetic field or temperature gradients leads to an amplification of the asymmetries.

5. We construct magnetograms, $M$, as (minus) the Stokes $V$ signal of Fe I $630.25 \mathrm{~nm}$ at $\Delta \lambda=+10$ pm from line center. $M$ provides a rough estimate of the longitudinal magnetic flux (Landi degl'Innocenti 1992).

\section{Identification, morphology, and proper motions of ECs}

In Figs. 3 and 4 we show the temporal evolution of the centerside penumbra and the moat region of AR 10781 during the 156 min covered by the June 30 data set. From top to bottom, the panels display the continuum intensity at $1565 \mathrm{~nm}$, the Doppler velocities calculated from the Stokes $V$ zero-crossing shifts of Fe I $1565.28 \mathrm{~nm}$ and Fe I $630.25 \mathrm{~nm}^{2}$, and the logarithm of the total linear polarization of Fe I $1564.85 \mathrm{~nm}$ and Fe I $630.25 \mathrm{~nm}$.

We identify ECs as structures of enhanced velocity signal which propagate outward across the penumbra. During the

2 The velocity is computed only for pixels exhibiting normal twolobed $V$ profiles with amplitudes greater than $0.2 \%$ in Fe I $630.25 \mathrm{~nm}$ and $0.5 \%$ in Fe I $1564.85 \mathrm{~nm}$.
Table 4. Phenomenological properties of the observed ECs. Those reaching the quiet photosphere are marked in bold face. Parentheses indicate ECs that leave the FOV or appear outside of it. The date and time of appearance of each EC are specified in the second and third columns. The fourth column displays the radial distance at which the EC is seen for the first time. Only the values of the ECs appearing inside the FOV are given. The fifth column displays the time each EC stays in the penumbra. The seventh column shows the propagation velocity, corrected for the viewing angle. The errors are the standard deviations in the determination of the proper motions. The last two columns display the maximum lengths and widths attained by each EC, corrected for projection effects.

\begin{tabular}{|c|c|c|c|c|c|c|c|c|}
\hline$\overline{\mathrm{EC}}$ & Date & $\begin{array}{c}t_{\text {app }} \\
\text { [min] }\end{array}$ & $r_{\text {app }}$ & $\begin{array}{c}\tau \\
{[\mathrm{min}]}\end{array}$ & Filament & $\begin{array}{c}v_{\text {prop }} \\
{\left[\mathrm{km} \mathrm{s}^{-1}\right]}\end{array}$ & $\begin{array}{c}\text { Length } \\
{[\mathrm{km}]}\end{array}$ & $\begin{array}{l}\text { Width } \\
{[\mathrm{km}]}\end{array}$ \\
\hline $\bar{A}$ & $06 / 30$ & 3.9 & 0.4 & 15.6 & 2 & $2.7 \pm 0.5$ & 2700 & 1000 \\
\hline (B) & $06 / 30$ & 15.6 & 0.5 & & 2 & $2.9 \pm 0.0$ & 500 & 400 \\
\hline $\mathrm{C}$ & $06 / 30$ & 23.4 & 0.4 & 11.7 & 2 & $2.2 \pm 0.7$ & 2600 & 800 \\
\hline D & $06 / 30$ & 42.9 & 0.5 & 11.9 & 2 & $3.3 \pm 0.3$ & 2300 & 900 \\
\hline E & $06 / 30$ & 46.8 & 0.5 & 11.9 & 1 & $.1 \pm 0.4$ & 1700 & 800 \\
\hline $\mathbf{F}$ & $06 / 30$ & 54.8 & 0.4 & 39.0 & 2 & $1.7 \pm$ & 3000 & 1200 \\
\hline G & $06 / 30$ & 86.0 & 0.6 & 11.7 & 1 & $3.7 \pm$ & 1700 & 500 \\
\hline (H) & $06 / 30$ & 113.5 & 0.7 & & 3 & $1.7 \pm 0.0$ & 1000 & 500 \\
\hline (I) & $06 / 30$ & 129.1 & & & 1 & $1.5 \pm 0.4$ & 2000 & 800 \\
\hline $\mathrm{J}$ & $06 / 30$ & 136.9 & 0.7 & 3.9 & 3 & $3.5 \pm 0.0$ & 1500 & 800 \\
\hline K & $06 / 30$ & 144.7 & 0.5 & 7.8 & 1 & $2.1 \pm 0.6$ & 1700 & 800 \\
\hline (L) & 07/01 & 5.9 & 0.7 & & & $2.2 \pm 0.8$ & 1200 & 600 \\
\hline M & $07 / 01$ & 9.9 & 0.7 & 7.9 & & $4.2 \pm 0.6$ & 1800 & 800 \\
\hline$(\mathbf{N})$ & 07/01 & 23.8 & & & & $2.0 \pm 0.2$ & 1200 & 500 \\
\hline (O) & 07/01 & 61.6 & 0.8 & & & $1.4 \pm 0.5$ & 1100 & 600 \\
\hline Mear & & & 0.6 & $\overline{13.5}$ & & 2.6 & 1700 & $\overline{700}$ \\
\hline
\end{tabular}

236 min of our observations, fifteen ECs appear in the midpenumbra and then migrate to the outer penumbral boundary. They are observed in the two spectral ranges simultaneously. We have outlined them with contours of different colors and letters in Figs. 3 and 4. The boundaries of the ECs have been defined as isocontours of specific values of the Doppler velocity, hence they should be considered as approximate.

To quantify the radial distances where the ECs appear, we first calculate the temporal average of the penumbral borders. They are used to define the normalized penumbral radial distance, $r$, such that $r=0$ represents the umbra/penumbra boundary and $r=1$ the outer edge of the spot.

Table 4 summarizes some phenomenological properties of the observed ECs. The radial distances where they appear, $r_{\text {app }}$, vary from 0.4 to 0.7 with an average of 0.6. Their lifetimes, $\tau$, inside the penumbra range from 3.9 to $39 \mathrm{~min}$ with an average of $13.5 \mathrm{~min}^{3}$. ECs can be classified in two different groups: (a) those that disappear at the outer penumbral boundary, hereafter type I ECs; and (b) those that reach the quiet photosphere and enter the moat (D, F, M, and N), type II in the following. Most of the ECs belong to the first group.

The propagation velocities of the ECs inside the penumbra, corrected for the viewing angle, range from $1.4 \mathrm{~km} \mathrm{~s}^{-1}$ to $4.2 \mathrm{~km} \mathrm{~s}^{-1}$ with an average of $2.6 \mathrm{~km} \mathrm{~s}^{-1}$ (seventh column of Table 4). These velocities are in rough agreement with values reported earlier (Shine et al. 1994; Rimmele 1994; Georgakilas \& Christopoulou 2003). To compute the propagation velocity of a given EC we have fitted straight lines to the $(x, y)$ positions of its edge farther from the umbra as a function of time. The slopes of these lines give the apparent propagation velocities along the

\footnotetext{
${ }^{3} \tau$ is only calculated for the ECs that do not leave the FOV.
} 


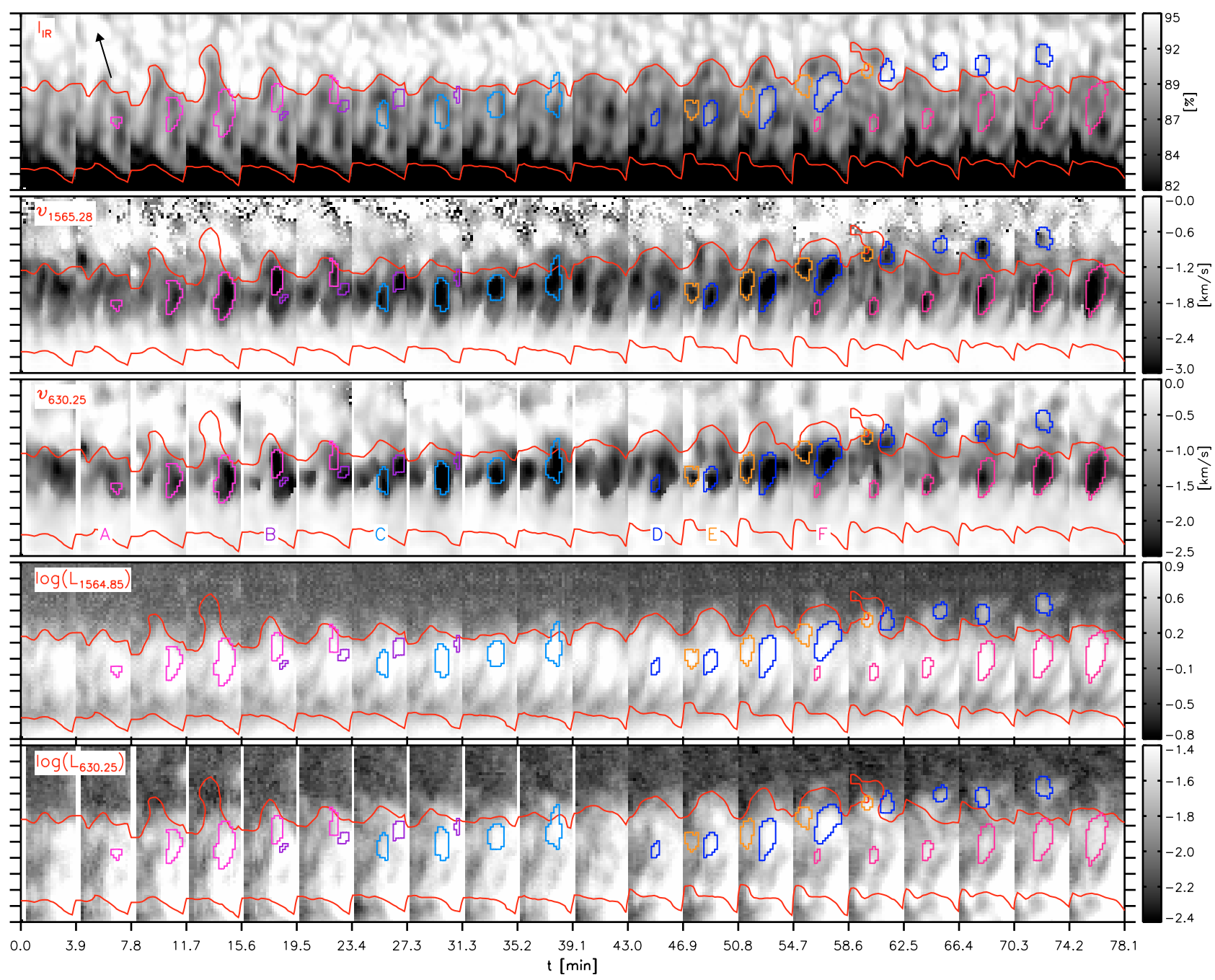

Fig. 3. Evolution of line parameters in the center-side penumbra of AR 10781 on June 30. Top to bottom: Continuum intensity at $1565 \mathrm{~nm}$, Stokes $V$ zero-crossing velocity of Fe I $1565.28 \mathrm{~nm}$ and Fe I $630.25 \mathrm{~nm}$, logarithm of the total linear polarization of Fe I $1564.85 \mathrm{~nm}$ and Fe I $630.25 \mathrm{~nm}$. Color contours outline the ECs. The letters at the bottom of the third panel label each EC. Red lines indicate the inner and outer penumbral boundaries. Tickmarks in the $y$-axis represent $1^{\prime \prime}$. The arrow points to disk center. $t=0$ min corresponds to June 30, 8:47 UT.

line of symmetry ${ }^{4}(\mathrm{LS})$ and perpendicularly to it. Due to projection effects the distances are contracted along the LS. We have corrected for this assuming that ECs move parallel to the solar surface: the vertical motion of ECs must be very small, otherwise they would leave the line forming region in a few minutes ${ }^{5}$.

In our observations, most ECs appear as elongated structures in the radial direction, and their sizes usually increase as they migrate outwards. To quantify the typical sizes of the ECs we define a major and a minor axis for each one. The major axis is the segment connecting the edges of the EC along its longer direction. The minor axis is perpendicular to the major axis and passes through its midpoint. We compute the length and width of the ECs along these axes and correct them for projection effects. The maximum length varies from 500 to $3000 \mathrm{~km}$ with an average of $1700 \mathrm{~km}$, while the maximum width ranges from 400 to

${ }^{4}$ The line of symmetry is the projection of the LOS onto the solar surface. In the case of sunspots, it coincides with the line connecting the sunspot center and the disk center.

5 The thickness of the photosphere is around $500 \mathrm{~km}$. If the vertical motions were greater than $600 \mathrm{~m} \mathrm{~s}^{-1}$, ECs departing from continuum forming layers would cross the entire photosphere in less than their mean lifetime $(\sim 13.5 \mathrm{~min})$.
$1200 \mathrm{~km}$ with an average of $700 \mathrm{~km}$ (cf. the two last columns of Table 4). For these computations we use only the ECs that are not partially outside the FOV. In Fig. 5 we represent the maximum, minimum, and mean sizes of the individual ECs. This figure also demonstrates that the lengths are usually greater than the widths.

Shine et al. (1994) described the ECs as cloud-like structures roughly $1000 \mathrm{~km}$ in length. The radial spacing between subsequent ECs was about $2000 \mathrm{~km}$. Our lengths are slightly larger than those reported by Shine et al. (1994), while the typical separation between two consecutive ECs is around $500 \mathrm{~km}$ in our observations (cf. the second panel of Fig. 3 at $t=15.6$ and $t=54.7 \mathrm{~min})$.

\section{Relation with penumbral filaments}

The second, third, and fourth panels of Figs. 3 and 4 demonstrate that ECs move outward following penumbral filaments with larger Doppler velocities and linear polarization signals than their surroundings. At least three different penumbral filaments can be distinguished in the June 30 data set. One of them is visible in the middle of the maps during the whole time sequence. The other two are partially outside of the FOV. We label the left-side, center, and right-side filaments as \#1, \#2, and \#3, 


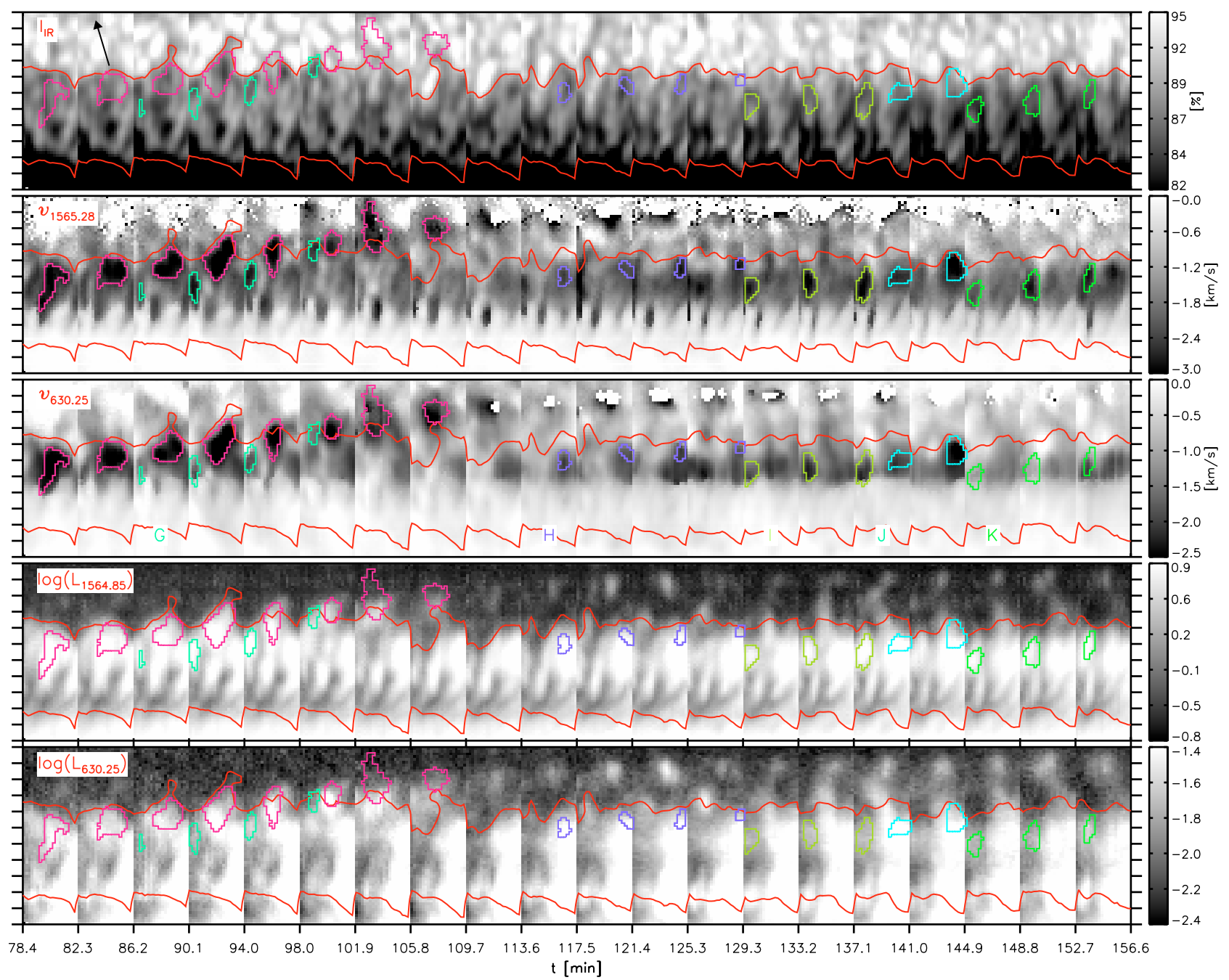

Fig. 4. Same as Fig. 3, from $t=78.4$ to $t=156.6 \mathrm{~min}$.

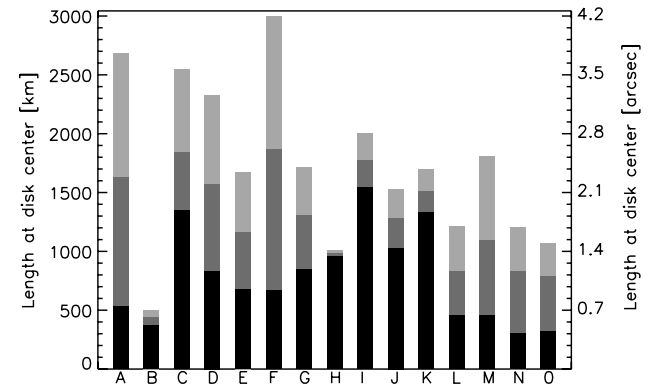

Evershed cloud

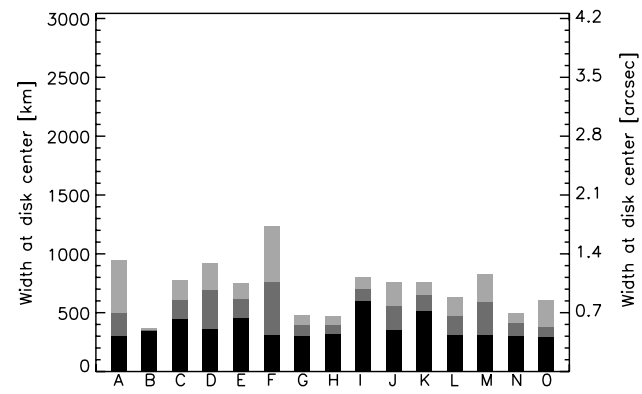

Evershed cloud

Fig. 5. Maximum, mean, and minimum lengths (top) and widths (bottom) of the ECs, corrected for projection effects (light to dark gray). respectively. The sixth column of Table 4 identifies the filament along which the ECs propagate outward.

Figure 6 blows up the velocity and polarization maps of the penumbra at $t=93.8 \mathrm{~min}$. The various panels display Doppler velocities, total linear polarizations, and total circular polarizations, along with their ratio, $L / V$. Penumbral filaments are outlined with contours of different colors which have been constructed taking into account the velocity and linear polarization signals. As can be seen, the filaments appear as structures of enhanced $v, L$, and $L / V$, and their contrast is much higher in the quantities derived from the infrared lines. They exhibit larger circular polarization signals than their surroundings in $\mathrm{Fe} I$ $1564.85 \mathrm{~nm}$, but smaller signals in Fe I $630.25 \mathrm{~nm}$. These properties indicate that the filaments are penumbral intra-spines (Lites et al. 1993).

In Fig. 7 we show the linear polarization of Fe I $1564.85 \mathrm{~nm}$ as a function of time for the maps where EC A is detected. Pink and yellow contours outline EC A and filament \#2, respectively. While the shape of the filament changes with time, it always remains visible from the inner to the outer penumbral boundary. We note that the pixels with larger linear polarizations (marked with orange contours) coincide with the positions of EC A. This behavior is also observed in the other ECs. Hence, in addition to higher Doppler velocities, ECs display larger linear polarization signals than the intra-spines hosting them. 

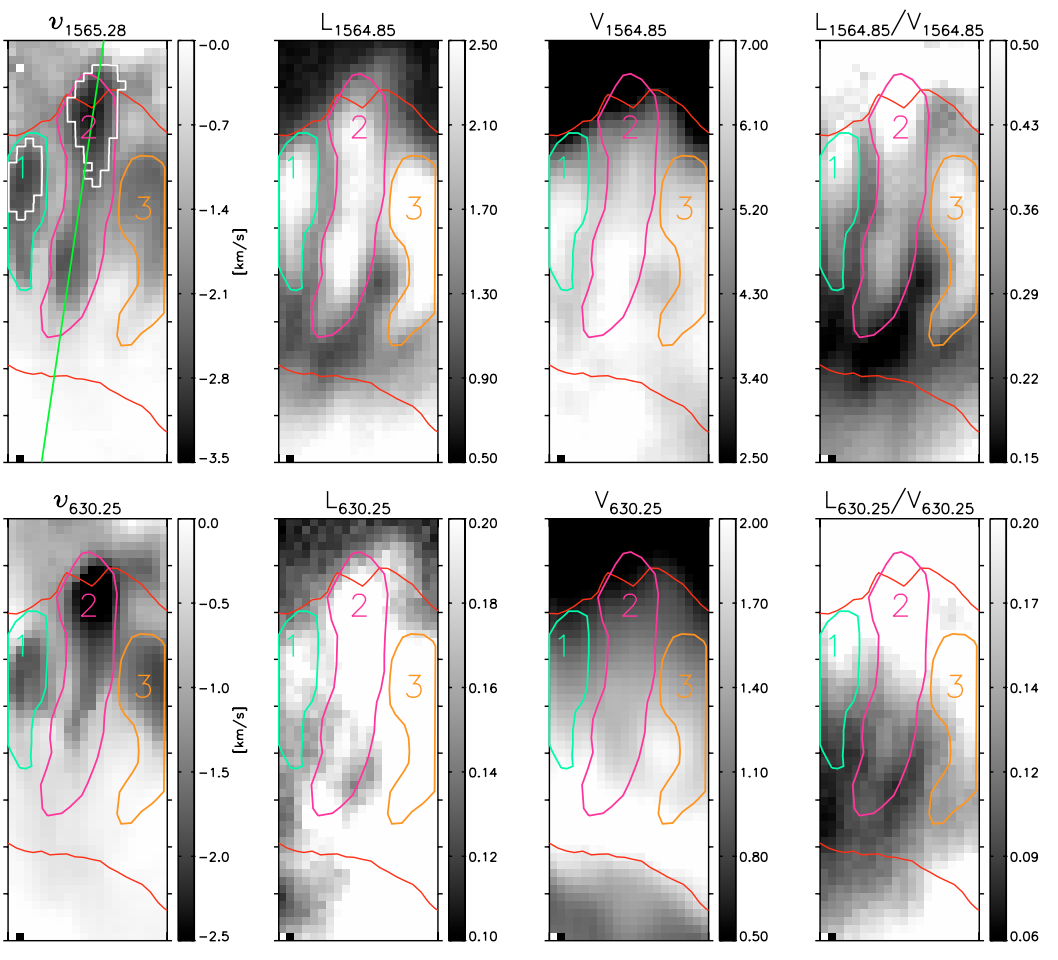

Fig. 6. From left to right: Maps of Stokes $V$ zerocrossing velocity for Fe I $1565.28 \mathrm{~nm}$ (top) and Fe I $630.25 \mathrm{~nm}$ (bottom), total linear polarization, total circular polarization, and $L / V$ ratio for Fe I $1564.85 \mathrm{~nm}$ (top) and Fe I $630.25 \mathrm{~nm}$ (bottom) at $t=93.8 \mathrm{~min}$. Color contours mark, and numbers label, three different penumbral filaments. White contours outline ECs $\mathrm{F}$ and $\mathrm{G}$. The green line represents a cut along filament \#2.

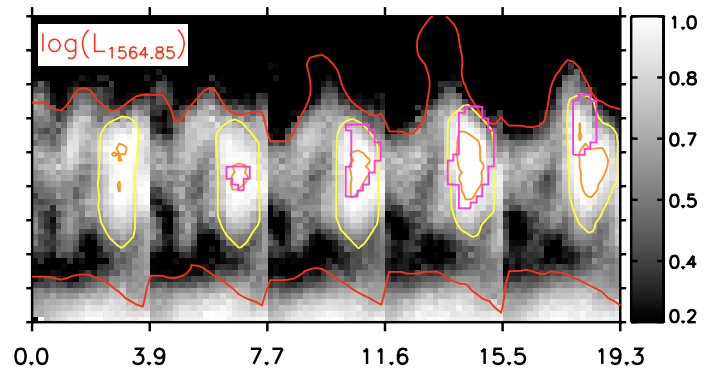

Fig. 7. Logarithm of the total linear polarization of Fe I $1564.85 \mathrm{~nm}$ as a function of time. Yellow and pink contours mark filament \#2 and EC A, respectively. Orange contours outline pixels with $\log L_{1564.85}>1$.

The polarization signals provide a rough estimate of the magnetic field inclination under some restrictive assumptions: if the pixel is occupied by a laterally homogeneous atmosphere, the lines are fully split, and the orientation of the magnetic field does not change with depth, a higher inclination to the LOS implies larger $L$ and smaller $V$ signals, i.e., larger $L / V$ ratios. Thus, Fig. 6 suggests that the intra-spines have more inclined magnetic fields than their surroundings, while Fig. 7 could indicate that the ECs are the most inclined structures inside them. However, the different behavior of the observables (in particular Stokes $V$ ) implies that no unambiguous interpretation is possible without more sophisticated analyses.

\section{Periodicity}

As already mentioned, the Evershed flow displays variations on time scales of 8-25 min. This quasi-periodic behavior is produced by ECs moving along intra-spines. The periodic times scales of $15 \mathrm{~min}$ are associated with the appearance (Shine et al. 1994) or appearance/disappearance (Rimmele 1994) of ECs in individual filaments. A precise estimation of the frequency of these processes requires a Fourier analysis of the data sets.
Looking at Figs. 3-4 it is obvious that the orientation of the filaments changes with time. Hence, one has to select different paths in each map to avoid the artificial peaks that contamination by spatial points outside the filament would induce in the power spectra. Given the difficulties, we do not pursue this kind of analysis here. However, a visual inspection of the maps provides valuable information about the periodic appearance of ECs.

In Figs. 3 and 4 one can observe a repetitive behavior associated with the appearance of ECs along filament \#2. Its time scale is about $15 \mathrm{~min}$. A cycle begins with EC A at $t=3.9 \mathrm{~min}$ and ends 15 min later when the EC arrives at the penumbral border. At that moment EC B appears in the mid penumbra, starting a new cycle. After EC B reaches the outer penumbral edge, EC C appears and another cycle begins. Subsequent cycles take place in filament \#2 with ECs D and F. This behavior is irregular and does not happen during the whole time sequence. For filament \#1 the periodic time scale seems to be of the order of $40 \mathrm{~min}$. This is the time gap between the appearance of ECs E, G, and I (third column of Table 4). Filament \#3 is visible during the second half of the sequence. Only two ECs are observed to follow this filament, and the time between their appearances is around $23 \mathrm{~min}$. Therefore, our observations suggest the existence of different time scales in different filaments. Longer time series mapping larger regions of the penumbra will be needed to confirm or discard this possibility.

\section{Spectropolarimetric properties of ECs}

Here we determine the typical Doppler velocities, $L / V$ ratios, and Stokes $V$ area asymmetries associated with ECs. To simplify the analysis, a single value of the parameters is calculated for each EC as follows. We compute the spatial average of the line parameters in all the maps where a given EC is observed, and then take the maximum of these average values.

Table 5 summarizes the results for the lines with higher sensitivity to magnetic fields (Fe I $1564.85 \mathrm{~nm}$ and Fe I $630.25 \mathrm{~nm}$ ). The maximum unsigned blueshifts are about $3.2 \mathrm{~km} \mathrm{~s}^{-1}$ and 
Table 5. Maximum Doppler shifts, linear-to-circular polarization ratios, and Stokes $V$ asymmetries attained by each EC. The second and third columns are the unsigned blueshifts of Fe I $1564.85 \mathrm{~nm}$ and Fe I $630.25 \mathrm{~nm}$. The fourth and fifth columns display the ratios of total linear and circular polarization of Fe I $1564.85 \mathrm{~nm}$ and Fe I $630.25 \mathrm{~nm}$. The last two columns are the area asymmetries of Fe I $1564.85 \mathrm{~nm}$ and Fe I $630.25 \mathrm{~nm}$. The errors represent the standard deviation of the individual parameters within each EC. The mean values of the observational parameters for the June 30 and July 1 data sets are marked in bold face.

\begin{tabular}{lcccccr}
\hline \hline EC & $\begin{array}{l}\left|v_{1564.85}\right| \\
{\left[\mathrm{km} \mathrm{s}^{-1}\right]}\end{array}$ & $\begin{array}{c}\left|v_{630.25}\right| \\
{\left[\mathrm{km} \mathrm{s}^{-1}\right]}\end{array}$ & $(L / V)_{1564.85}$ & $(L / V)_{630.25}$ & $\begin{array}{c}\delta A_{1564.85} \\
{[\%]}\end{array}$ & \multicolumn{1}{c}{$\begin{array}{c}\delta A_{630.25} \\
{[\%]}\end{array}$} \\
\hline A & $3.3 \pm 0.3$ & $2.4 \pm 0.2$ & $0.44 \pm 0.02$ & $0.19 \pm 0.03$ & $5.9 \pm 2.0$ & $2.4 \pm 2.3$ \\
B & $3.4 \pm 0.3$ & $2.5 \pm 0.3$ & $0.50 \pm 0.05$ & $0.27 \pm 0.02$ & $7.3 \pm 3.2$ & $7.7 \pm 1.8$ \\
C & $3.2 \pm 0.3$ & $2.6 \pm 0.3$ & $0.45 \pm 0.03$ & $0.20 \pm 0.03$ & $6.9 \pm 1.7$ & $4.2 \pm 3.3$ \\
D & $3.2 \pm 0.5$ & $2.4 \pm 0.5$ & $0.44 \pm 0.07$ & $0.22 \pm 0.04$ & $5.8 \pm 2.4$ & $5.1 \pm 2.8$ \\
E & $3.1 \pm 0.3$ & $2.1 \pm 0.4$ & $0.44 \pm 0.06$ & $0.27 \pm 0.02$ & $5.3 \pm 1.9$ & $7.0 \pm 3.7$ \\
F & $3.4 \pm 0.5$ & $2.5 \pm 0.4$ & $0.56 \pm 0.14$ & $0.29 \pm 0.05$ & $6.5 \pm 2.4$ & $9.6 \pm 6.5$ \\
G & $3.0 \pm 0.3$ & $1.5 \pm 0.1$ & $0.55 \pm 0.05$ & $0.27 \pm 0.03$ & $9.1 \pm 3.7$ & $10.1 \pm 1.6$ \\
H & $3.1 \pm 0.2$ & $1.9 \pm 0.3$ & $0.49 \pm 0.02$ & $0.24 \pm 0.01$ & $6.1 \pm 1.7$ & $5.8 \pm 2.5$ \\
I & $3.1 \pm 0.2$ & $1.9 \pm 0.2$ & $0.36 \pm 0.04$ & $0.14 \pm 0.02$ & $3.6 \pm 1.3$ & $-2.4 \pm 1.3$ \\
J & $3.2 \pm 0.3$ & $2.3 \pm 0.3$ & $0.49 \pm 0.02$ & $0.24 \pm 0.01$ & $5.5 \pm 2.6$ & $1.7 \pm 2.5$ \\
K & $3.1 \pm 0.2$ & $1.5 \pm 0.4$ & $0.41 \pm 0.05$ & $0.16 \pm 0.02$ & $3.2 \pm 1.5$ & $-3.2 \pm 2.3$ \\
\hline Mean & $\mathbf{3 . 2}$ & $\mathbf{2 . 2}$ & $\mathbf{0 . 4 7}$ & $\mathbf{0 . 2 2}$ & $\mathbf{5 . 9}$ & $\mathbf{4 . 4}$ \\
\hline L & $2.1 \pm 0.1$ & $1.4 \pm 0.1$ & $0.87 \pm 0.07$ & $0.37 \pm 0.03$ & $4.7 \pm 1.0$ & $-2.1 \pm 1.1$ \\
M & $2.2 \pm 0.1$ & $1.5 \pm 0.1$ & $0.83 \pm 0.06$ & $0.41 \pm 0.04$ & $5.5 \pm 1.4$ & $4.7 \pm 3.4$ \\
N & $2.1 \pm 0.2$ & $1.2 \pm 0.2$ & $0.83 \pm 0.08$ & $0.38 \pm 0.03$ & $5.9 \pm 2.5$ & $6.9 \pm 4.7$ \\
O & $1.9 \pm 0.3$ & $1.0 \pm 0.1$ & $0.69 \pm 0.02$ & $0.31 \pm 0.02$ & $5.4 \pm 2.7$ & $3.3 \pm 1.8$ \\
\hline Mean & $\mathbf{2 . 1}$ & $\mathbf{1 . 3}$ & $\mathbf{0 . 8 1}$ & $\mathbf{0 . 3 7}$ & $\mathbf{5 . 4}$ & $\mathbf{3 . 2}$ \\
\hline
\end{tabular}

$2.2 \mathrm{~km} \mathrm{~s}^{-1}$ for Fe I $1564.85 \mathrm{~nm}$ and Fe I $630.25 \mathrm{~nm}$ on June 30 , and $2.1 \mathrm{~km} \mathrm{~s}^{-1}$ and $1.3 \mathrm{~km} \mathrm{~s}^{-1}$ on July 1 . These velocities are lower than the values reported by Shine et al. (1994) and Rimmele (1994). The reason is that we calculate an average over a group of pixels, while they used velocities from individual pixels.

The mean linear-to-circular polarization ratios are of the order of 0.47 and 0.22 in Fe I $1564.85 \mathrm{~nm}$ and Fe I $630.25 \mathrm{~nm}$ for the June 30 observations, and around 0.81 and 0.37 for the July 1 data set. Since the ECs show much larger $L / V$ ratios in the infrared, vector magnetographs working at infrared wavelengths should detect them more easily than visible magnetographs.

The Stokes $V$ area asymmetry of Fe I $1564.85 \mathrm{~nm}, \delta A_{1564.85}$, amounts to $5.9 \%$ on June 30 and $5.4 \%$ on July 1 , while $\delta A_{630.25}$ is around $4.4 \%$ and $3.2 \%$ on June 30 and July 1 , respectively. For some ECs, the Fe I $630.25 \mathrm{~nm}$ area asymmetry is of the order of its standard deviation, suggesting that $\delta A_{630.25}$ has a strong dependence on radial distance.

The large variations of the Doppler shifts and $L / V$ ratios of the ECs as observed on two consecutive days are consistent with the idea that the flow and magnetic field are almost horizontal to the solar surface. The spot was nearer to disk center on July 1. Because of projection effects, highly inclined velocity and magnetic field vectors should produce smaller blueshifts, larger linear polarization signals, and smaller circular polarization signals (i.e., larger $L / V$ ratios) in the center-side penumbra as the spot approaches the disk center. This explains why the velocities are higher on June 30 while the $L / V$ ratios are larger on July 1 .

\section{Comparison of line parameters in ECs and intra-spines}

In a sunspot penumbra, the physical properties are known to vary radially from the umbra to the outer penumbral boundary. For this reason, we compare the observational properties of the ECs and the intra-spines as follows. We calculate mean line parameters at each radial distance for the pixels that form the ECs and normalize them to the mean parameters of the pixels inside the intra-spines that are at the same radial distance. This is done for each map separately. Figures 8 and 9 show histograms with the results of the analysis for Fe I $1564.8 \mathrm{~nm}$ and Fe I $630.25 \mathrm{~nm}$ on June 30 . The results for the July 1 data set are equivalent (not shown).

Interestingly, the ECs exhibit larger Doppler velocities and $L / V$ ratios than the penumbral intra-spines in both $\mathrm{Fe} \mathrm{I}$ $630.25 \mathrm{~nm}$ and Fe I $1564.85 \mathrm{~nm}$. Hence, our measurements suggest that ECs possess the most inclined magnetic fields of the penumbra. In Paper II we will examine this conjecture with the help of Stokes inversions.

The ECs also show larger Fe I $1564.85 \mathrm{~nm}$ area asymmetries than the intra-spines. This might be the result of stronger gradients of the physical quantities along the LOS and/or enhanced visibility of some magnetic components of the penumbra in the ECs. By contrast, we find smaller values of $\delta A_{630.25}$ in the ECs. This opposite behavior is surprising and could be due to the different heights of formation of the two spectral lines.

The relation between ECs and continuum intensity is not well established in the literature. Shine et al. (1994) and Rouppe van der Voort (2003) report that ECs are mainly correlated with locally bright continuum features, while Rimmele (1994) and Georgakilas \& Christopoulou (2003) do not find any correlation. By visual inspection alone it is difficult to determine whether ECs are bright or dark continuum features. In the intensity histogram of Fig. 9, the ECs seem to show a preference for locally dark continuum intensities at $630 \mathrm{~nm}$. A similar but less pronounced trend is observed in the $1565 \mathrm{~nm}$ continuum (Fig. 8). These results have to be taken with caution because of the existence of unresolved structures at the angular resolution of our observations $\left(\sim 0.6^{\prime \prime}-0.7^{\prime \prime}\right)$. In Fig. 10 we display co-spatial maps of speckle-reconstructed G-band intensity, continuum intensity at $630 \mathrm{~nm}$, and continuum intensity at $1565 \mathrm{~nm}$. The figure demonstrates that what we detect as dark/bright features in the spectropolarimetric data are indeed a mixture of both in the G-band filtergrams. As the ECs migrate outward, the dark/bright features inside them change, but in general the G-band intensities are locally darker at the position of the ECs. Spectropolarimetric observations at higher spatial 

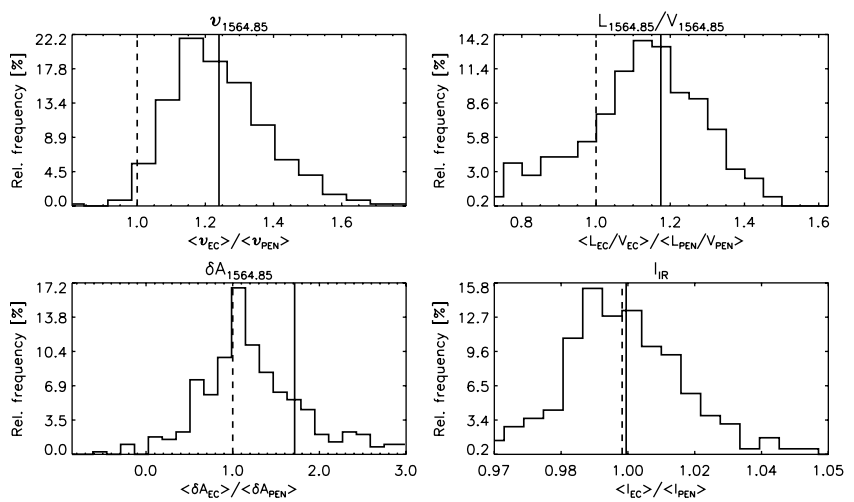

Fig. 8. Normalized distribution of the ratio of Doppler velocities $(v)$, linear-to-circular polarization signals $(L / V)$, Stokes $V$ area asymmetries $(\delta A)$, and continuum intensities $(I)$ in the ECs and the rest of pixels of the intra-spines located at the same radial distance. The line parameters are derived from Fe I $1564.85 \mathrm{~nm}$. The vertical solid lines represent the mean of the distributions. The histograms correspond to the June 30 data set.
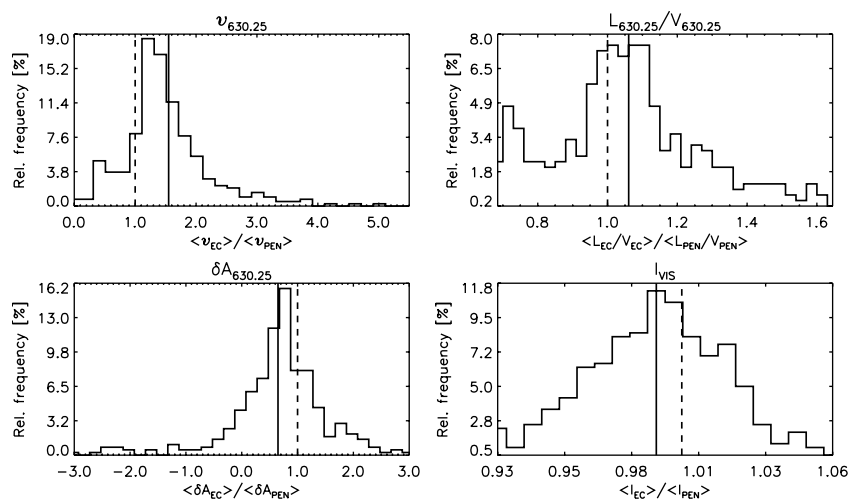

Fig. 9. Same as Fig. 8 for Fe I $630.25 \mathrm{~nm}$.

resolution are certainly needed to draw definite conclusions on the brightness of ECs.

Finally, we note that the histograms of Figs. 8 and 9 provide information about the average line parameters of the ECs independently of their position in the penumbra. These properties can change with radial distance. For example, Schlichenmaier et al. (2005) suggested that flow filaments link bright and dark continuum features at different radial distances. Since ECs move along intra-spines (i.e., flow filaments), we may expect them to be associated with locally bright features in the inner penumbra. This conjecture will be examined in Sect. 10.

\section{Dependence of Doppler velocities on height}

The magnitude of the Doppler velocity depends on the spectral line used to compute it (cf. the first two columns of Table 5). Figure 11 shows the maximum unsigned Doppler velocities of the ECs inferred from each line. There we see that the velocities indicated by the infrared lines are the largest ones. The IR lines of the set are formed deeper than the others. This implies that the Evershed flow is more conspicuous in deep atmospheric layers, in agreement with previous findings (Maltby 1964; Balthasar et al. 1997; Westendorp Plaza et al. 2001; Mathew et al. 2003; Schlichenmaier et al. 2004; Bellot Rubio et al. 2006). The different velocities deduced from different spectral lines may be the result of (a) a change of the modulus of the velocity vector with
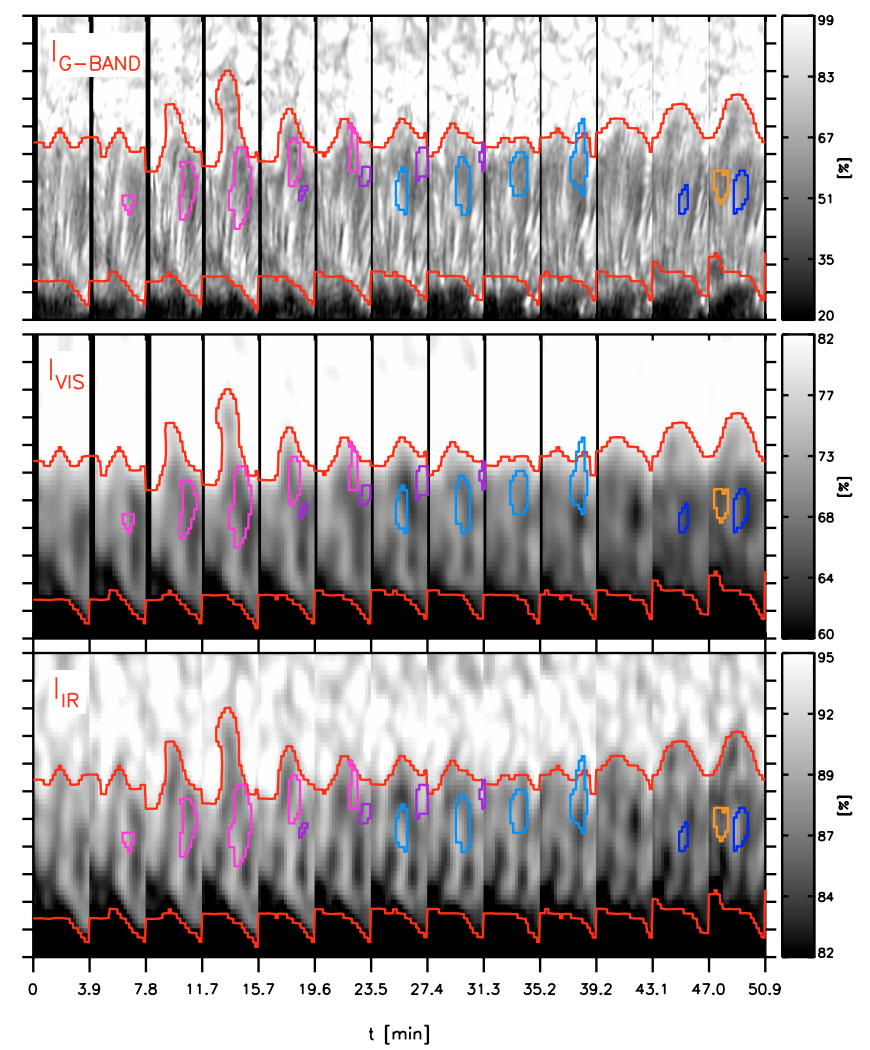

Fig. 10. From top to bottom: Cospatial speckle-reconstructed G-band filtergrams, continuum intensity maps at $630 \mathrm{~nm}$, and continuum intensity maps at $1565 \mathrm{~nm}$. Color contours outline the ECs.

height; (b) a change of the inclination of the velocity vector with height; or (c) a combination of both effects.

\section{Proper motions vs Doppler velocities}

It is of interest to compare the proper motions of the ECs along the LOS (calculated as the motion along the LS multiplied by the sine of the heliocentric angle) with their maximum Doppler velocities. If the blueshifts detected in the ECs of the centerside penumbra are produced by proper motions, both quantities should be similar in magnitude.

Figure 12 plots the LOS projection of the propagation velocities of the ECs vs the maximum Doppler velocities inferred from Fe I $1564.85 \mathrm{~nm}$ and Fe I $630.25 \mathrm{~nm}$. The horizontal bars indicate the standard deviation of the Doppler velocities within each contour, and the vertical bars the errors in the determination of propagation velocities. The observed LOS velocities are systematically higher than the LOS component of the proper motion. This result agrees with the findings of Shine et al. (1994) and Rimmele (1994) and suggests that the Evershed flow cannot only be the manifestation of the radial propagation of ECs across the penumbra. The observed behavior is also compatible with the sea-serpent scenario described by Schlichenmaier (2002), in which magnetic flux loops slowly migrate outward with high flow velocity inside them.

\section{Evolution of line parameters with radial distance}

In Sect. 7 we compared the line parameters of ECs and intraspines disregarding the fact that there is a radial variation of the physical conditions in the penumbra. Here we investigate how 


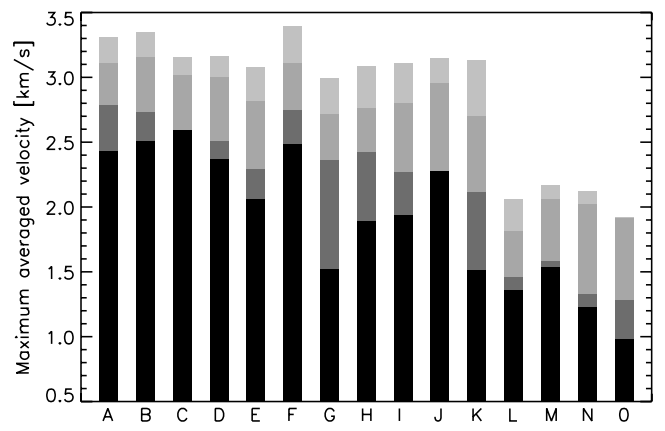

Evershed cloud

Fig. 11. Maximum Doppler velocities of the observed ECs as derived from the Stokes $V$ zero-crossing wavelengths. Maximum blueshifts for Fe I 1564.85 nm, Fe I 1565.28 nm, Ti I 630.38 nm, and Fe I 630.25 nm are represented with different shades of gray, from light to dark. Note that the blueshifts are unsigned.
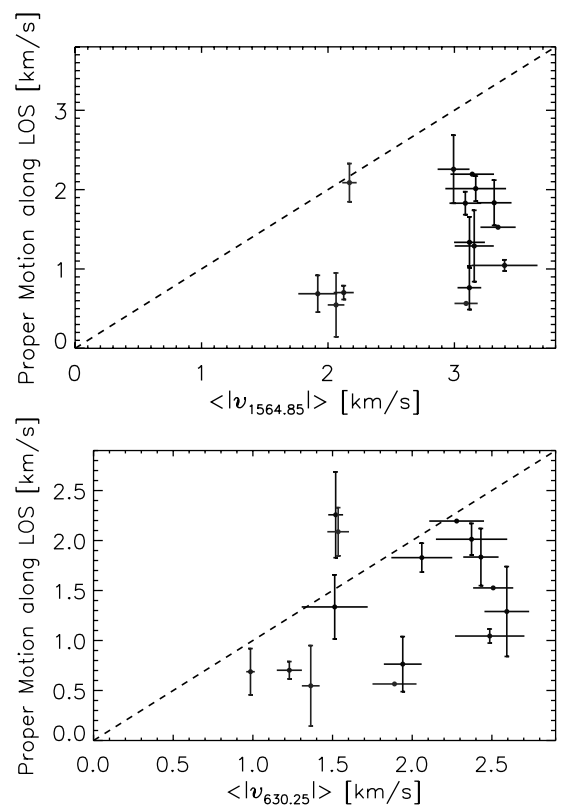

Fig. 12. Proper motions along the LOS vs maximum Doppler velocities from Fe I $1565.28 \mathrm{~nm}$ (top) and Fe I $630.25 \mathrm{~nm}$ (bottom) for the different ECs. Circles represent the maximum average Doppler velocities. The horizontal bars represent their standard deviations. The vertical bars are the errors in the determination of proper motions.

the line parameters change with radial distance. To that aim, we average the parameters of pixels inside the ECs at the same radial distance, and construct a curve of their variation with $r$. We note that those curves represent the average evolution of the ECs in the penumbra. Similar curves for the pixels of the intra-spines (excluding the ECs) are calculated to provide a basis for comparison.

Figures 13 and 14 show the results for Fe I $1564.85 \mathrm{~nm}$ and Fe I $630.25 \mathrm{~nm}$, respectively. We find larger Doppler velocities in the ECs at all radial distances. Also interesting is the different shape of the velocity curves. The velocity always increases in the ECs as they migrate outward, from $v_{630.25}=-1.8 \mathrm{~km} \mathrm{~s}^{-1}$ and $v_{1564.85}=-2.8 \mathrm{~km} \mathrm{~s}^{-1}$ at $r=0.5$ to $v_{630.25}=-2.2 \mathrm{~km} \mathrm{~s}^{-1}$ and $v_{1564.85}=-3.0 \mathrm{~km} \mathrm{~s}^{-1}$ at $r=1$. In the intra-spines, however, the Doppler velocity slightly decreases beyond $r \sim 0.6$.

The ratio of linear-to-circular polarization is larger in the ECs at all distances, which is more obvious for the IR line. In addition, both the ECs and the intra-spines display larger $L / V$
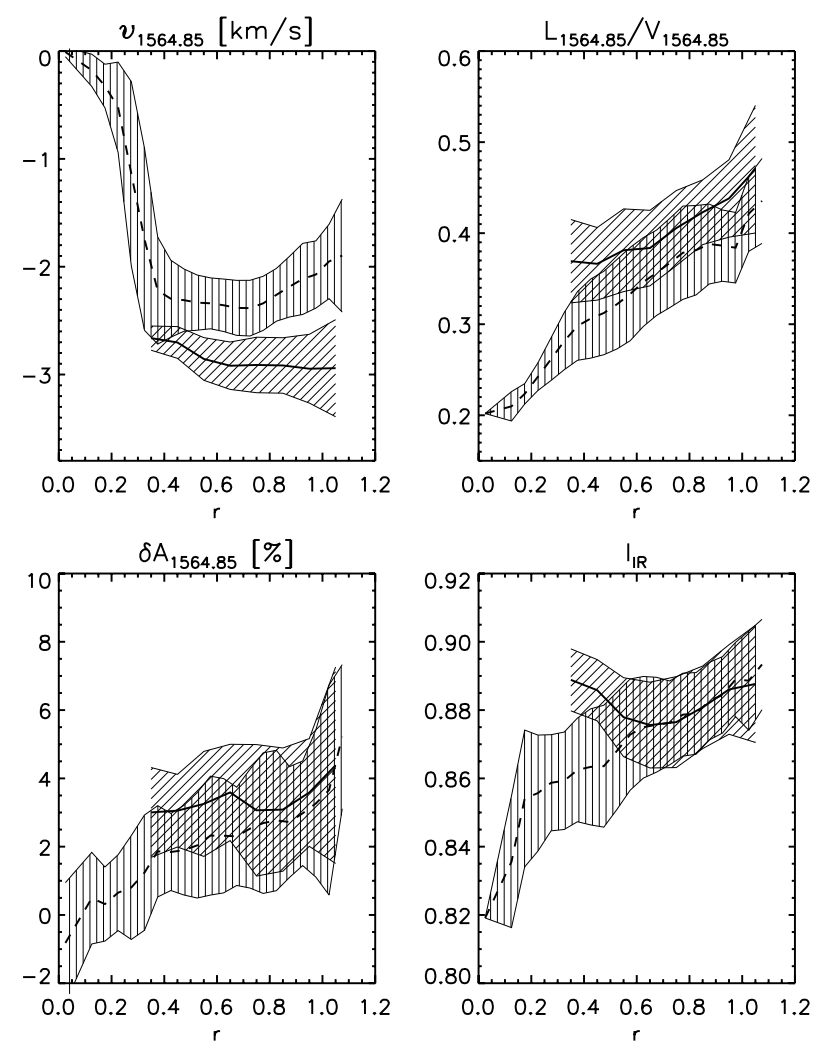

Fig. 13. Average Stokes $V$ zero-crossing velocity, ratio of the total linear and circular polarization, area asymmetry, and continuum intensity of Fe I $1564.85 \mathrm{~nm}$ (top) for pixels inside the ECs (solid line) and the rest of pixels of the intra-spines (dashed line) as a function of radial distance, for the time sequence observed on June 30. Shaded areas indicate the standard deviations around the mean value.

values at larger radial distances. For the ECs, the ratio increases from $(L / V)_{630.25}=0.14$ and $(L / V)_{1564.85}=0.38$ at $r=0.5$ up to $(L / V)_{630.25}=0.24$ and $(L / V)_{1564.85}=0.47$ at $r=1$.

The area asymmetry $\delta A$ of the two lines also increases monotonically with radial distance, showing larger values in the ECs than in the intra-spines. The area asymmetries change from $\delta A_{630.25}=-1.5 \%$ and $\delta A_{1564.85}=4.5 \%$ at $r=0.5$ to $\delta A_{630.25}=6.2 \%$ and $\delta A_{1564.85}=7.2 \%$ at $r=1$.

The continuum intensity curves display an interesting variation with radial distance. In the ECs, both $I_{\mathrm{IR}}$ and $I_{\mathrm{VIS}}$ are greater than the intra-spine average at the radial distances where they appear $(r \sim 0.4)$. From the mid to the outer penumbra, however, the continuum intensities of ECs and intra-spines do not exhibit significantly different values.

\section{Disappearance of type I ECs}

Most of the type I ECs observed on June 30 disappear suddenly in the penumbra, near the edge of the spot. This indicates that the time they take to vanish is usually shorter than the cadence of the observations (3.9 min on June 30 ). There is, however, an interesting case where a type I EC is seen to decay.

Figure 15 shows the evolution of the Doppler velocities of EC A as derived from Fe I $1565.28 \mathrm{~nm}$. The EC has vanished at $t=23.4 \mathrm{~min}$ (there is no dark patch where one would expect to find it, just outside of the penumbra). To quantify the evolution of the velocities we choose radial cuts passing through the middle of the EC (cf. the the vertical solid lines in Fig. 15). The variation of the Doppler velocities along the cuts is shown in 

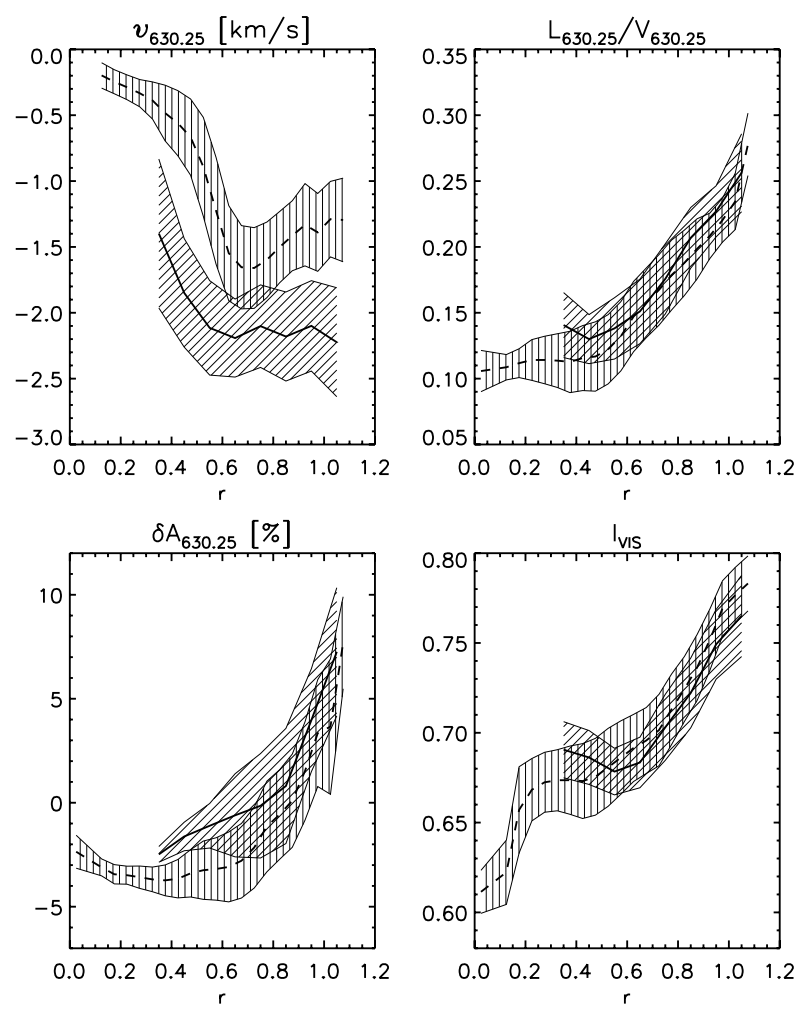

Fig. 14. Same as Fig. 13 for the quantities inferred from Fe I $630.25 \mathrm{~nm}$.

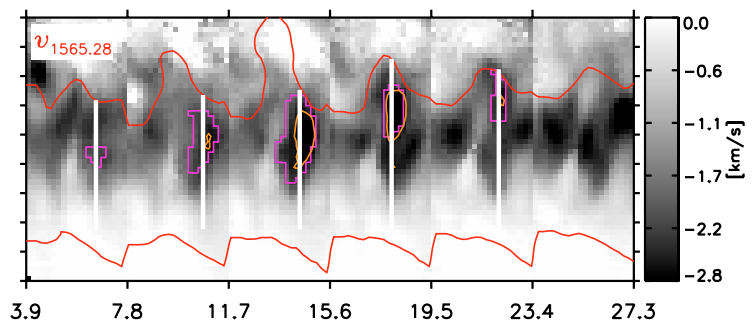

Fig. 15. Maps of Stokes $V$ zero-crossing velocity of Fe I $1565.28 \mathrm{~nm}$ as a function of time. Pink contours mark EC A. Orange contours enclose pixels with $v_{630.25}$ and $v_{1565.28}$ lower than $-2.3 \mathrm{~km} \mathrm{~s}^{-1}$ and $-2.7 \mathrm{~km} \mathrm{~s}^{-1}$, respectively. The vertical lines mark a radial cut passing through the EC. Each tickmark in the $y$-axis represents $1 "$.

Fig. 16. Colors represent different times and the horizontal lines mark the upper and lower edges of the EC. In agreement with the behavior indicated by Figs. 13 and 14, the EC velocities increase with radial distance until $t=15.6 \mathrm{~min}$. Then, a strong drop in the velocities occurs at $t=19.5 \mathrm{~min}$, after which the EC is no longer seen. EC C behaves in a similar way, so the process just described seems to be common for type I ECs.

\section{Evolution of type II ECs beyond the outer penumbral boundary}

Shine et al. (1994) and Rimmele (1994) reported that some ECs penetrate slightly beyond the outer penumbral boundary before dissolving. On June 30 and July 1, four ECs crossed the edge of AR 10781. Here we describe the evolution of these type II ECs in the sunspot moat.

\subsection{Modification of the outer edge of the penumbra by ECs}

ECs D and F moved along filament \#2 (see Fig. 6). Figure 17 displays the temporal evolution of the Doppler velocity, the continuum intensity, and the linear polarization in the filament as a function of radial distance. The three signals are strongly correlated near the outer penumbral boundary, which becomes more evident when an EC reaches the quiet photosphere. This happens at $t=58.7 \mathrm{~min}$ for EC D and $t=97.7 \mathrm{~min}$ for EC F. When an EC penetrates into the quiet photosphere, the outer penumbra does the same: the velocity and linear polarization increase and the continuum intensity decreases just outside the spot, i.e., the penumbra grows at that position. Such a behavior has been reported earlier by Shine et al. (1994), but only for the intensity and velocity signals.

\subsection{Disappearance of type /I ECs in the moat}

The evolution of ECs D and F in the sunspot moat can be followed in Figs. 5 and 6. EC D (pink contours) crossed the penumbral boundary between $t=93.8$ and $t=97.7 \mathrm{~min}$. Its proper motion outside the spot was $\sim 1.9 \mathrm{~km} \mathrm{~s}^{-1}$. Anomalous Stokes $V$ profiles appear where one would locate the EC at $t=109.6 \mathrm{~min}$. We cannot determine Doppler velocities from those profiles. However, the EC is easily detected in the velocity maps computed from the Doppler shifts of Stokes $I$ (not shown). The evolution of EC F can be tracked until $t=109.6$ min. Thus, EC F disappeared as a velocity structure 11.9 min after crossing the spot boundary.

The evolution of ECs M and N outside the spot is shown in Figs. B.1 and B.2. EC M is only visible in two maps before it leaves the FOV. EC N disappeared 2'. 1 from the spot boundary.

The phenomenological properties of ECs D, F, M, and $\mathrm{N}$ in the moat are summarized in Table 6. The behavior of type II ECs outside the spot is very similar: they quickly dissolve $(\sim 13.8 \mathrm{~min})$ without reaching large radial distances from the outer penumbral boundary ( 1'.9). Inside the spot ECs show up as elongated structures, but type II ECs adopt a roundish shape when they enter the moat. Moreover, their proper motions are reduced relative to those they exhibit in the penumbra (compare Tables 6 and 4).

As described by Cabrera Solana et al. (2006), ECs D and F became moving magnetic features (MMFs) in the sunspot moat. This suggests that type II ECs are the precursors of at least some MMFs. The relation between MMFs and the magnetic field of the penumbra has been studied by Sainz Dalda \& Martínez Pillet (2005), Ravindra (2006), Kubo et al. (2007), and Cabrera Solana et al. (2008).

\section{Summary and conclusions}

We have presented a detailed description of the temporal evolution of the Evershed flow including, for the first time, its polarization signatures. Our main findings can be summarized as follows:

1. We identify fifteen Evershed clouds (ECs) that appear in the mid penumbra. They propagate with speeds of $\sim 2.6 \mathrm{~km} \mathrm{~s}^{-1}$ along penumbral filaments characterized by larger values of the linear polarization, linear-to-circular polarization ratios, and Doppler velocities than their surroundings. These filaments can be associated with the penumbral intra-spines described by Lites et al. (1993). 

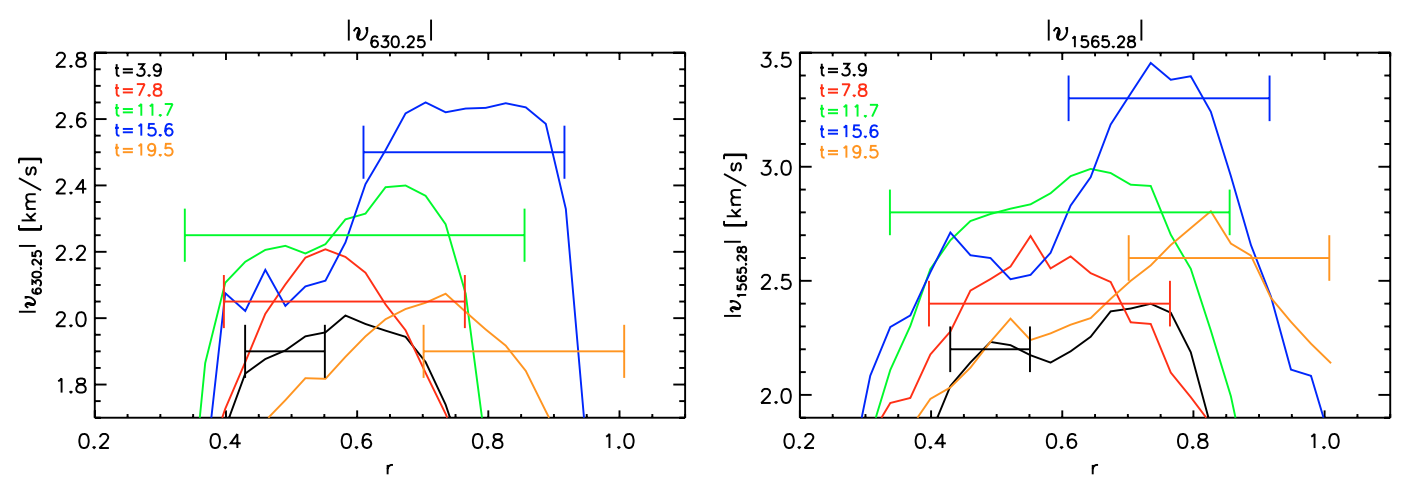

Fig. 16. Variation of $\left|v_{630.25}\right|$ and $\left|v_{1565.28}\right|$ along the radial cuts shown in Fig. 15. The colors indicate different times and the horizontal lines mark the upper and lower edges of EC A.
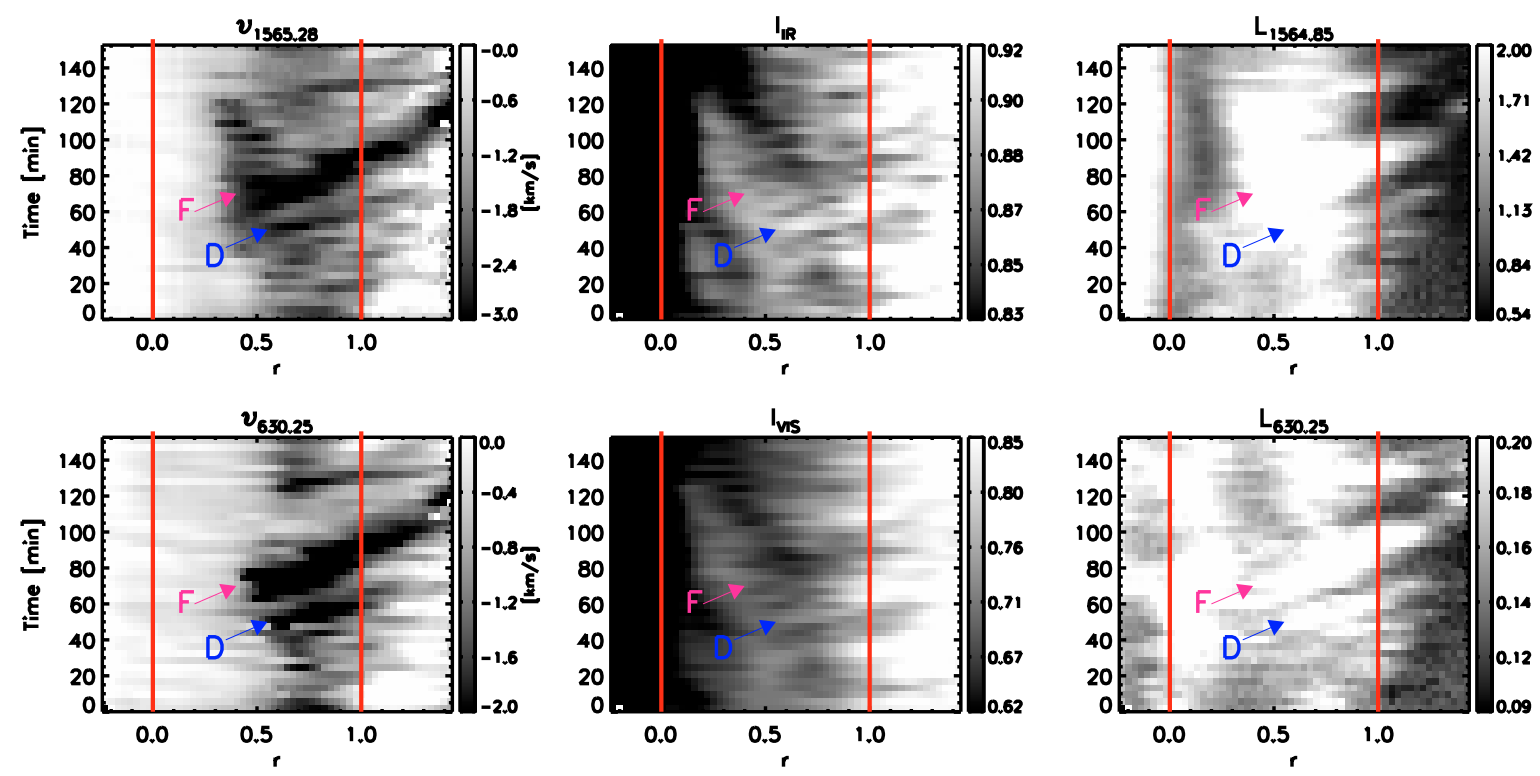

Fig. 17. Temporal evolution of line parameters along intra-spine \#2 (cf. the green radial cut in the first panel of Fig. 6). Top: Stokes $V$ zero-crossing velocity of Fe I $1565.28 \mathrm{~nm}$ (left), continuum intensity at $1565 \mathrm{~nm}$ (center), and total linear polarization of Fe I $1564.85 \mathrm{~nm}$ (right). Bottom: Stokes $V$ zero-crossing velocity of Fe I $630.25 \mathrm{~nm}$ (left), continuum intensity at $630.2 \mathrm{~nm}$ (center), and total linear polarization of Fe I $630.25 \mathrm{~nm}$ (right). The $x$-axis gives the average penumbral radial distance and the $y$-axis displays time. The arrows indicate the positions of ECs D and F.

2. ECs can be classified in two types: (a) ECs that disappear in the outer penumbra (type I); and (b) ECs that cross the visible border of the spot and enter the sunspot moat (type II). Most of the observed ECs belong to type I.

3. The motion of ECs in the penumbra is essentially radial.

4. ECs have greater lengths $(\sim 1700 \mathrm{~km})$ than widths $(\sim 700 \mathrm{~km})$, and their sizes increase as they migrate outward.

5. Our observations suggest that different time scales associated with the appearance of ECs exist. We find periodic behaviors of the order of $15 \mathrm{~min}$ and $40 \mathrm{~min}$ in two different intraspines.

6. ECs display larger Doppler velocities and $L / V$ ratios in the infrared lines of the set. This implies that: (a) ECs are more conspicuous in deeper atmospheric layers; and (b) vector magnetographs working in the infrared should detect them more easily.

7. ECs exhibit larger Doppler velocities, $L / V$ ratios, and area asymmetries than the intra-spines hosting them. This might imply that the magnetic and dynamic configuration of the intra-spines are modified by the passage of ECs. Assuming that the velocity and magnetic field vectors are aligned (Bellot Rubio et al. 2003), the enhanced Doppler velocities of the ECs can only be produced by an increase in the modulus of the velocity vector.
Table 6. Phenomenological properties of type II ECs in the moat. The time and distance from the outer penumbral boundary where the ECs vanish as velocity structures are given in the second and third columns. The fourth column displays the time each of them survived in the quiet photosphere. The proper motions of the ECs in the moat are given in the fifth column. The last two columns display their maximum lengths and widths, corrected for LOS effects, outside the penumbra. The parentheses indicate that EC M leaves the FOV before disappearing.

\begin{tabular}{lrcccrr}
\hline \hline EC & $\begin{array}{c}t_{\text {dis }} \\
{[\mathrm{min}]}\end{array}$ & $\begin{array}{r}d_{\text {dis }} \\
{\left[{ }^{\prime \prime}\right]}\end{array}$ & $\begin{array}{c}\tau_{\text {ph }} \\
{[\mathrm{min}]}\end{array}$ & $\begin{array}{c}v_{\text {prop }} \\
{\left[\mathrm{km} \mathrm{s}^{-1}\right]}\end{array}$ & $\begin{array}{r}\text { Length } \\
{[\mathrm{km}]}\end{array}$ & $\begin{array}{r}\text { Width } \\
{[\mathrm{km}]}\end{array}$ \\
\hline $\mathrm{D}$ & 74.3 & 1.8 & 15.6 & $0.6 \pm 0.3$ & 1300 & 700 \\
$\mathrm{~F}$ & 109.6 & 1.8 & 11.9 & $1.9 \pm 0.4$ & 1000 & 1200 \\
$(\mathrm{M})$ & & & & $3.5 \pm 0.0$ & 900 & 400 \\
$\mathrm{~N}$ & 57.8 & 2.1 & 14.0 & $1.3 \pm 0.1$ & 900 & 600 \\
\hline Mean & & 1.9 & 13.8 & 1.8 & 1000 & 700 \\
\hline
\end{tabular}

8. The Doppler velocities observed in ECs are larger when the sunspot is farther from disk center, while their $L / V$ ratios are smaller. These results are consistent with the idea that the flow and the magnetic field are almost horizontal to the solar surface.

9. ECs are brighter than intra-spines in the inner penumbra. The brightness excess is no longer seen in the mid and outer penumbra. 
10. The Doppler velocities of the ECs are systematically greater than the LOS projection of their proper motions, assumed parallel to the solar surface.

11. The blueshifts, linear-to-circular polarization ratios, and area asymmetries of the ECs increase with radial distance. In the intra-spines, $L / V$ and $\delta A$ also increase towards the edge of the spot, but the velocity decreases from the middle to the outer penumbra.

12. The disappearance time scales of type I ECs are shorter than the cadence of our observations ( $\sim 4 \mathrm{~min})$.

13. The outer penumbral boundary is modified by the passage of the ECs that reach the quiet photosphere. When a type II EC penetrates into the moat, the outer penumbral boundary does the same and the intensity decreases in the quiet photosphere.

14. Four ECs are seen to cross the visible border of the spot. Once they enter the moat: (a) their propagation velocities are reduced (1.8 vs. $2.6 \mathrm{~km} \mathrm{~s}^{-1}$ ); (b) they adopt a roundish shape; and (c) their blueshifts quickly decrease until they vanish. Type II ECs can be detected as velocity structures in the quiet photosphere for only about 14 min after crossing the outer penumbral boundary. They disappear relatively close to the $\operatorname{spot}\left(\sim 2^{\prime \prime}\right)$.

15. Some type II ECs become moving magnetic features in the sunspot moat (Cabrera Solana et al. 2006), but they do not show significant Doppler shifts.

The line parameters derived from the polarization profiles give us valuable information about the phenomenological properties of the ECs. However, the interpretation of these observational parameters in terms of physical quantities is not straightforward. For example, a larger value of the Doppler shift can be associated with a stronger Evershed flow, but also with a magnetic field which is less inclined to the LOS.

To remove these ambiguities, we have performed detailed Stokes inversions of the observed spectra. The results of the inversions, presented in Paper II of this series, will allow us to investigate the nature of the EC phenomenon.

Acknowledgements. This work has been supported by the Spanish MEC under project ESP2006-13030-C06-02 and Programa Ramón y Cajal. The German VTT is operated by the Kiepenheuer-Institut für Sonnenphysik at the Observatorio del Teide of the Instituto de Astrofísica de Canarias. The DOT is operated by Utrecht University at the Observatorio del Roque de Los Muchachos on La Palma, also of the Instituto de Astrofísica de Canarias.

\section{References}

Allende Prieto, C., Asplund, M., \& Fabiani Bendicho, P. 2004, A\&A, 423, 1109 Auer, L. H., \& Heasley, J. N. 1978, A\&A, 64, 67

Balthasar, H., Schmidt, W., \& Wiehr, E. 1997, Sol. Phys., 171, 331

Beck, C., Schlichenmaier, R., Collados, M., Bellot Rubio, L., \& Kentischer, T. 2005a, A\&A, 443, 1047
Beck, C., Schmidt, W., Kentischer, T., \& Elmore, D. 2005b, A\&A, 437, 1159 Beck, C., Bellot Rubio, L., Schlichenmaier, R., \& Sütterlin, P. 2007, A\&A, 472, 607

Bellot Rubio, L. R., Balthasar, H., Collados, M., \& Schlichenmaier, R. 2003, A\&A, 403, L47

Bellot Rubio, L. R., Schlichenmaier, R., \& Tritschler, A. 2006, A\&A, 453, 1117 Borrero, J. M., Bellot Rubio, L. R., Barklem, P. S., \& del Toro Iniesta, J. C. 2003, A\&A, 404, 749

Cabrera Solana, D. 2007, Ph.D. Thesis, University of Granada, Spain

Cabrera Solana, D., Bellot Rubio, L. R., \& del Toro Iniesta, J. C. 2005, A\&A, 439, 687

Cabrera Solana, D., Bellot Rubio, L. R., Beck, C., \& del Toro Iniesta, J. C. 2006, ApJ, 649, L41

Cabrera Solana, D., Bellot Rubio, L. R., Borrero, J. M., \& del Toro Iniesta, J. C. 2007, A\&A, submitted (Paper II)

Cabrera Solana, D., Bellot Rubio, L. R., Beck, C., \& del Toro Iniesta, J. C. 2008, $\mathrm{A} \& \mathrm{~A}$, in preparation

Collados, M., Rodríguez Hidalgo, I., Bellot Rubio, L., Ruiz Cobo, B., \& Soltau, D. 1999, in Astronomische Gesellschaft Meeting Abstracts, ed. R. E. Schielicke, 13

Collados, M. V. 2003, in Polarimetry in Astronomy, ed. S. Fineschi, SPIE, 4843, 55

del Toro Iniesta, J. C., Tarbell, T. D., \& Ruiz Cobo, B. 1994, ApJ, 436, 400

Georgakilas, A. A., \& Christopoulou, E. B. 2003, ApJ, 584, 509

Kubo, M., Shimizu, T., \& Tsuneta, S. 2007, ApJ, 659, 812

Landi degl'Innocenti, E. 1992, Magnetic field measurements (Solar Observations: Techniques and Interpretation), 71

Lites, B. W., Elmore, D. F., Seagraves, P., \& Skumanich, A. P. 1993, ApJ, 418, 928

Maltby, P. 1964, Astrophysica Norvegica, 8, 205

Martínez Pillet, V., Collados, M., Sánchez Almeida, J., et al. 1999, in High Resolution Solar Physics: Theory, Observations, and Techniques, ed. T. R. Rimmele, K. S. Balasubramaniam, \& R. R. Radick, ASP Conf. Ser. 183, 264 Mathew, S. K., Lagg, A., Solanki, S. K., et al. 2003, A\&A, 410, 695

Nave, G., Johansson, S., Learner, R. C. M., Thorne, A. P., \& Brault, J. W. 1994, ApJS, 94, 221

Pierce, A. K., \& Breckenridge, J. B. 1974, The Kitt Peak table of photographic solar spectrum wavelengths (Kitt Peak National Observatory Contribution, Tucson: Kitt Peak National Observatory, 1973-1974)

Ravindra, B. 2006, Sol. Phys., 237, 297

Reardon, K. P. 2006, Sol. Phys., 239, 503

Rezaei, R., Schlichenmaier, R., Beck, C., \& Bellot Rubio, L. R. 2006, A\&A, 454, 975

Rimmele, T. R. 1994, A\&A, 290, 972

Rouppe van der Voort, L. H. M. 2003, A\&A, 397, 757

Sainz Dalda, A., \& Martínez Pillet, V. 2005, ApJ, 632, 1176

Sánchez Almeida, J., \& Lites, B. W. 1992, ApJ, 398, 359

Schlichenmaier, R. 2002, Astron. Nachr., 323, 303

Schlichenmaier, R., \& Collados, M. 2002, A\&A, 381, 668

Schlichenmaier, R., Bellot Rubio, L. R., \& Tritschler, A. 2004, A\&A, 415, 731

Schlichenmaier, R., Bellot Rubio, L. R., \& Tritschler, A. 2005, Astron. Nachr., 326, 301

Schmidt, W., Beck, C., Kentischer, T., Elmore, D., \& Lites, B. 2003, Astron. Nachr., 324, 300

Shine, R. A., Title, A. M., Tarbell, T. D., et al. 1994, ApJ, 430, 413

Socas-Navarro, H., Pillet, V. M., Sobotka, M., \& Vázquez, M. 2004, ApJ, 614, 448

Solanki, S. K. 1993, Space Sci. Rev., 63,

Solanki, S. K. 2003, A\&ARv, 11, 153

Soltau, D., Berkefeld, T., von der Lühe, O., Wöger, F., \& Schelenz, T. 2002, Astron. Nachr., 323, 236

Westendorp Plaza, C., del Toro Iniesta, J. C., Ruiz Cobo, B., \& Pillet, V. M. 2001, ApJ, 547, 1148 
D. Cabrera Solana et al.: Temporal evolution of the Evershed flow in sunspots. I., Online Material $p 1$

\section{Online Material}




\section{Appendix A: Removal of scattered light}

In Fig. A.1 we present fits to the disk-center profiles of $\mathrm{Fe} \mathbf{I}$ $630.15 \mathrm{~nm}$ and $630.25 \mathrm{~nm}$ recorded on July 1, 2005 using Eq. (1). As can be seen, the two visible lines are well reproduced. The merit function of the fit (right panel) shows a minimum for $K \simeq 15 \%$ and $\sigma \simeq 6 \mathrm{pm}$. The same results are obtained for the profiles measured on June 30, 2005.

Figure A. 2 shows an example of the effect of the scattered light correction on the polarization spectra: the original Stokes $U$ profile displays signatures of the telluric lines, but they disappear after the correction.

\section{Appendix B: Line parameter maps from the July 1} observations 
D. Cabrera Solana et al.: Temporal evolution of the Evershed flow in sunspots. I., Online Material p 3
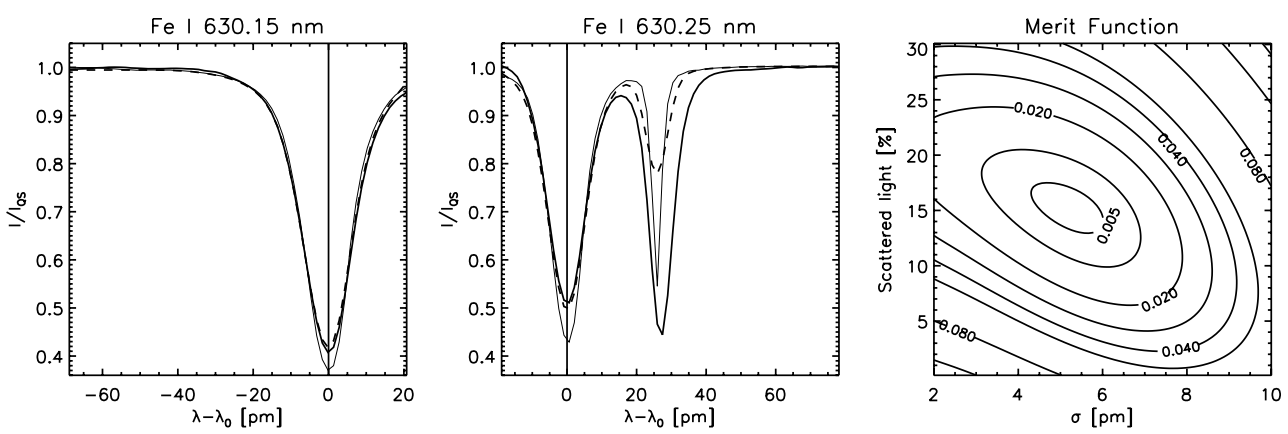

Fig. A.1. Comparison of the spectra at disk center of Fe I $630.15 \mathrm{~nm}$ (left panel) and Fe I $630.25 \mathrm{~nm}$ (center panel) as observed with FTS (thin solid) and POLIS (thick solid) for the 1 July dataset. The dashed lines are the best fits obtained by combining the FTS profiles with the fraction of scattered light and the resolving power of POLIS as explained in the text. The merit function of the fit is shown in the right panel. Note that the $\mathrm{O}_{2}$ telluric line is not considered in the fit.
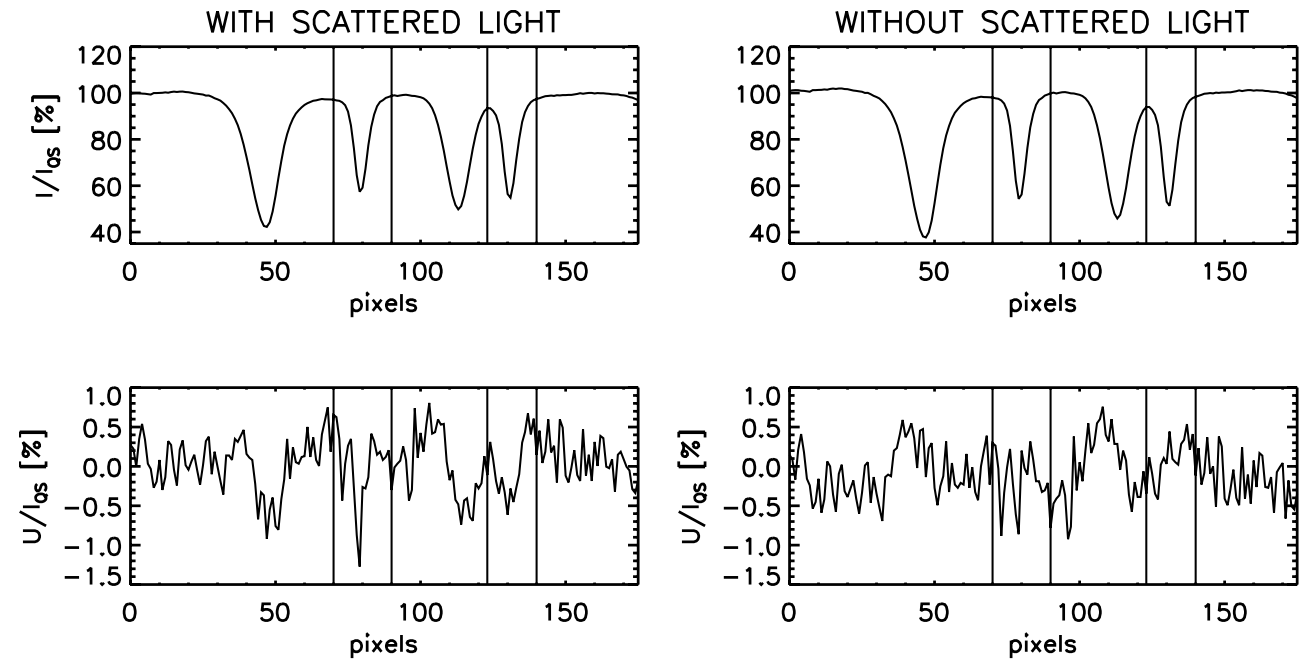

Fig. A.2. Stokes $I$ (top) and Stokes $U$ (bottom) profiles of the lines at $630 \mathrm{~nm}$ before (left) and after (right) correction for scattered light. The vertical lines indicate the positions of the $\mathrm{O}_{2}$ telluric lines. 
D. Cabrera Solana et al.: Temporal evolution of the Evershed flow in sunspots. I., Online Material p 4
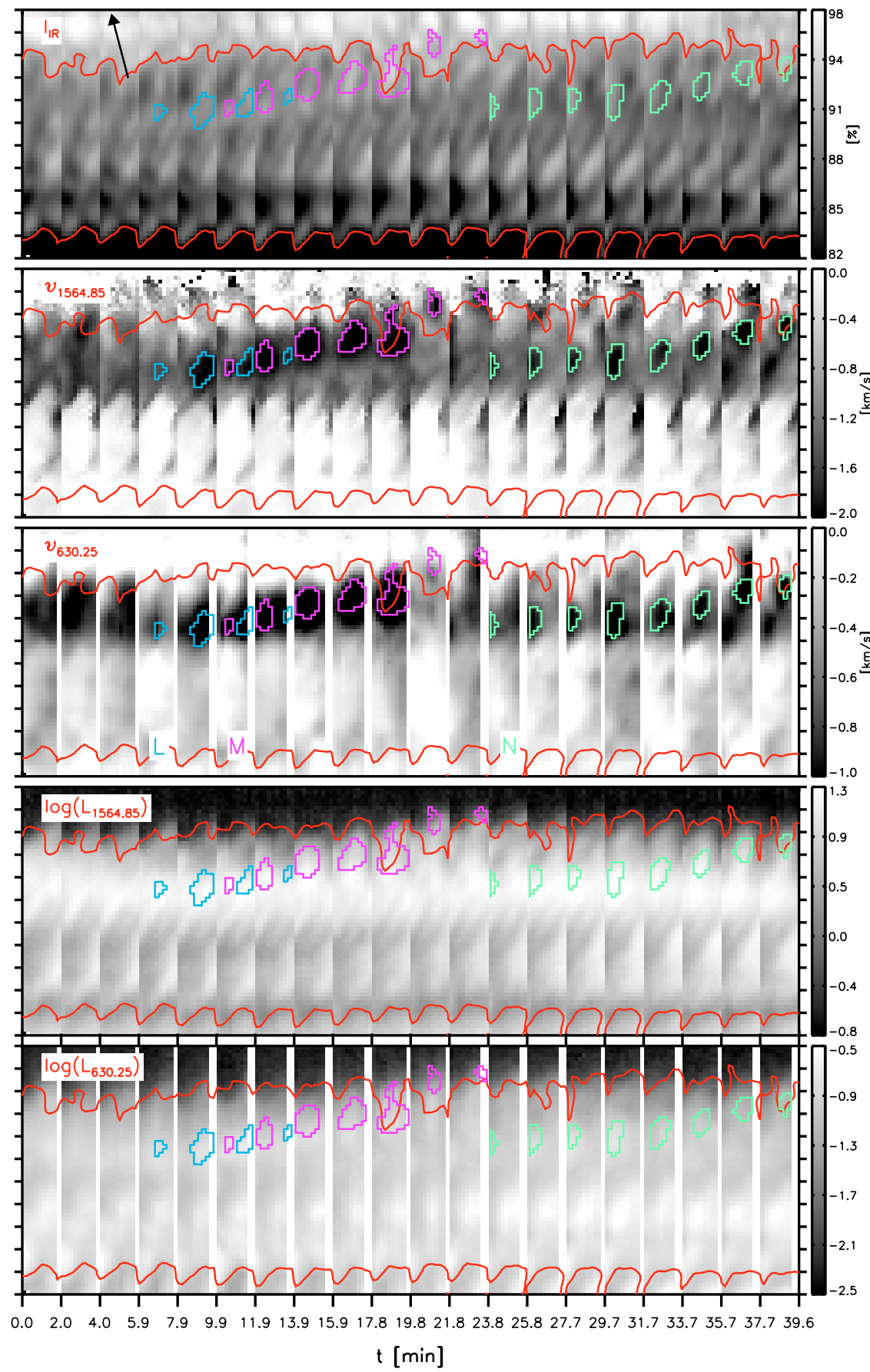

Fig. B.1. Time evolution of line parameters in the center-side penumbra of AR 10781 on July 1, 2005. From top to bottom: Continuum intensity at $1565 \mathrm{~nm}$, Stokes $V$ zero-crossing velocity of Fe I $1564.85 \mathrm{~nm}$ and Fe I $630.25 \mathrm{~nm}$, logarithm of the total linear polarization of Fe I $1564.85 \mathrm{~nm}$ and Fe I $630.25 \mathrm{~nm}$. Color contours outline the ECs. The letters at the bottom of the third panel label each EC. Red lines indicate the inner and outer penumbral boundaries. Each tickmark in the $y$-axis represent $1^{\prime \prime}$. The arrow marks the direction to disk center. 
D. Cabrera Solana et al.: Temporal evolution of the Evershed flow in sunspots. I., Online Material p 5
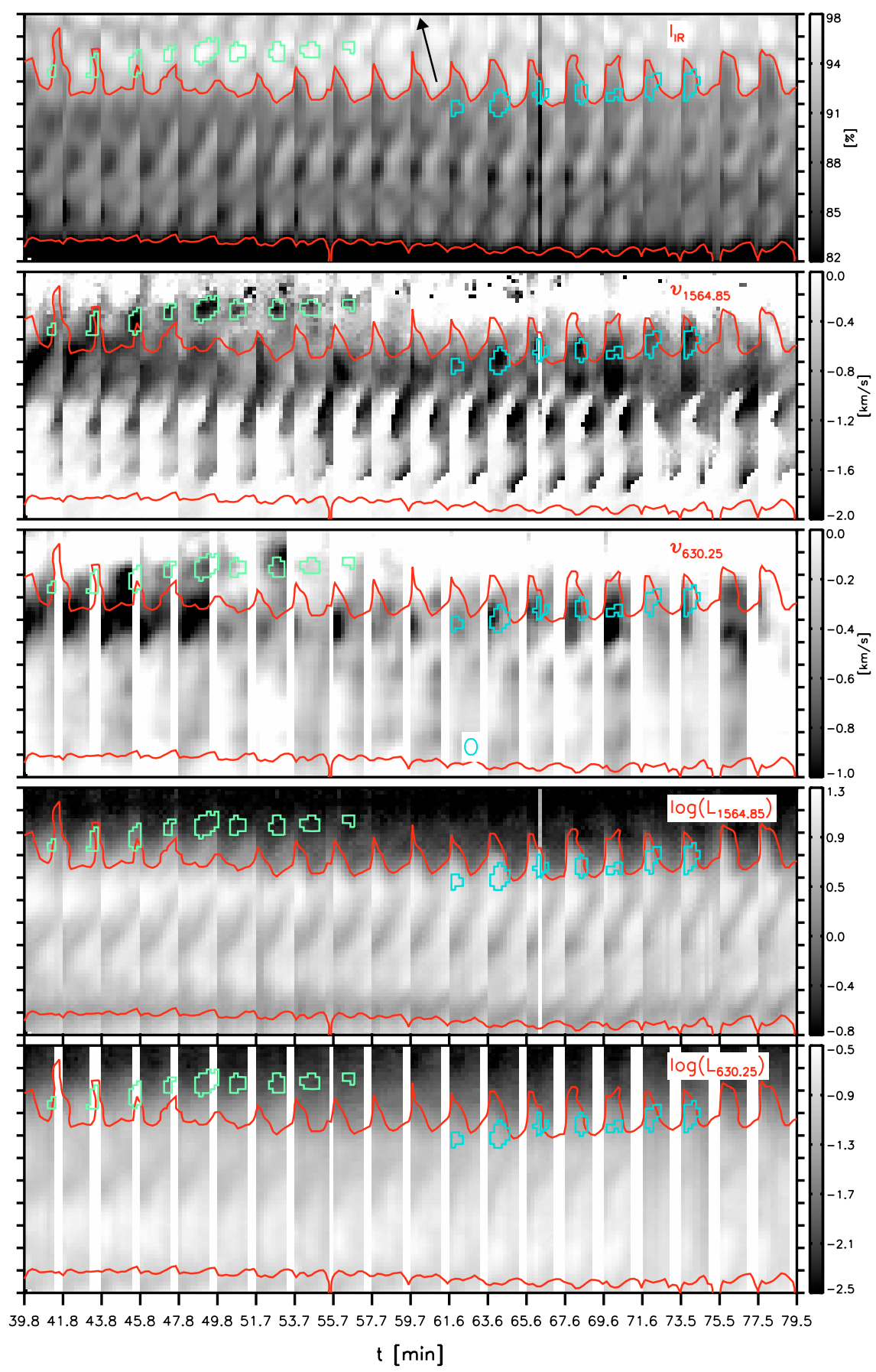

Fig. B.2. Same as Fig. B.1, from $t=39.8$ to $t=79.5 \mathrm{~min}$. 